\title{
Long-term evolution of electron distribution function due to nonlinear resonant interaction with whistler mode waves
}

\author{
Anton V. Artemyev ${ }^{1,2, \dagger}$, Anatoly I. Neishtadt ${ }^{2,3}$, Alexei A. Vasiliev ${ }^{2}$ \\ and Didier Mourenas ${ }^{4}$ \\ ${ }^{1}$ Institute of Geophysics and Planetary Physics, University of California, Los Angeles, California, USA \\ ${ }^{2}$ Space Research Institute, Moscow, Russia \\ ${ }^{3}$ Department of Mathematical Sciences, Loughborough University, Loughborough LE11 3TU, UK \\ ${ }^{4}$ CEA, DAM, DIF, Arpajon, France
}

(Received 19 December 2017; revised 13 March 2018; accepted 14 March 2018)

Accurately modelling and forecasting of the dynamics of the Earth's radiation belts with the available computer resources represents an important challenge that still requires significant advances in the theoretical plasma physics field of wave-particle resonant interaction. Energetic electron acceleration or scattering into the Earth's atmosphere are essentially controlled by their resonances with electromagnetic whistler mode waves. The quasi-linear diffusion equation describes well this resonant interaction for low intensity waves. During the last decade, however, spacecraft observations in the radiation belts have revealed a large number of whistler mode waves with sufficiently high intensity to interact with electrons in the nonlinear regime. A kinetic equation including such nonlinear wave-particle interactions and describing the long-term evolution of the electron distribution is the focus of the present paper. Using the Hamiltonian theory of resonant phenomena, we describe individual electron resonance with an intense coherent whistler mode wave. The derived characteristics of such a resonance are incorporated into a generalized kinetic equation which includes non-local transport in energy space. This transport is produced by resonant electron trapping and nonlinear acceleration. We describe the methods allowing the construction of nonlinear resonant terms in the kinetic equation and discuss possible applications of this equation.

Key words: wave-particle interaction

\section{Introduction}

Wave-particle resonant interaction plays an essential role for energy redistribution in a collisionless plasma, where this interaction is responsible for the relaxation of unstable particle distributions and the generation of high-energy particles. One important example of such systems is the Earth's radiation belts, where resonances between intense whistler mode waves and energetic electrons can result in electron

$\dagger$ Email address for correspondence: aartemyev@igpp.ucla.edu 


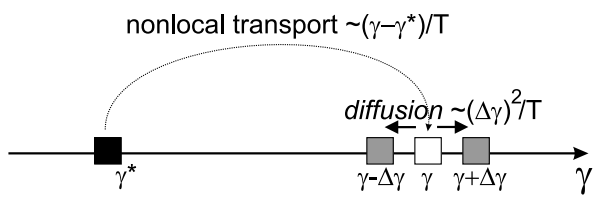

FIGURE 1. Scheme of particle energy $\gamma$ change over a time $T$ due to diffusion and due to non-local transport.

precipitation into the Earth atmosphere (e.g. Thorne et al. 2010) and acceleration to ultra-relativistic energies (e.g. Thorne et al. 2013). Numerical modelling of this interaction is based on a kinetic equation for the electron distribution function. For low intensity waves, the resonant interaction results in particle diffusion in velocity space, and the kinetic equation can be rewritten in the form of a diffusion equation (e.g. Schulz \& Lanzerotti 1974; Lyons \& Williams 1984). The corresponding diffusion coefficients can be calculated within the quasi-linear theory (Kennel \& Engelmann 1966; Trakhtengerts 1966). This approach is widely applied for simulations of the radiation belts dynamics (e.g. Albert \& Young 2005; Su, Zheng \& Wang 2010; Glauert, Horne \& Meredith 2014; Shprits et al. 2015). However, the diffusive approximation becomes inapplicable for sufficiently intense waves (Karpman 1974; Shapiro \& Sagdeev 1997; Tao et al. 2012a) which can interact nonlinearly with electrons. The main property of such a nonlinear interaction is that it is prolonged and very efficient, i.e. trapped particles remain trapped into resonance for a relatively long time and their energy can be significantly modified during a single resonant interaction (e.g. Solovev \& Shklyar 1986, and references therein). The final energy gain after such trapped acceleration depends on the wave characteristics and background magnetic field configuration and has been investigated in various conditions (see, e.g. Demekhov et al. 2006; Omura, Furuya \& Summers 2007; Summers \& Omura 2007; Bortnik, Thorne \& Inan 2008; Yoon, Pandey \& Lee 2013). Spacecraft observations in the Earth radiation belts have demonstrated the presence of a large number of intense whistler mode waves (Cattell et al. 2008; Cully, Bonnell \& Ergun 2008; Wilson et al. 2011; Agapitov et al. 2014; Santolík et al. 2014) which can nonlinearly resonate with electrons and lead to their trapping-induced acceleration.

Theoretical models describing nonlinear electron interaction with whistler mode waves are well developed for test particle trajectories (Nunn 1971, 1974; Karpman, Istomin \& Shklyar 1974). The inclusion of nonlinear effects in the kinetic equation for the electron distribution function can be performed using non-local operators acting on the distribution (Shklyar 1981; Artemyev et al. 2014; Omura et al. 2015). Such operators model the non-local relationships between different parts of the distribution in energy space: the particle distribution for a particular energy $\gamma$ depends not only on its close surrounding, but also on the distribution at energy $\gamma^{*}$ of trapped particles released from the resonance with energy $\gamma$ (see scheme in figure 1). Although, there are several examples of these non-local operators derived numerically (Omura et al. 2015) or analytically (Artemyev et al. 2014), there is so far no unified approach for the derivation of a kinetic equation including all nonlinear effects. Recently, such an approach was proposed for simple one-dimensional systems with electrostatic waves (Artemyev et al. 2017b) and we generalize it in the present paper to electromagnetic whistler mode waves propagating at an arbitrary angle relative to the background magnetic field. 
The paper is organized as follows: $\S 2.1$ is devoted to general expressions of whistler mode wave electromagnetic fields through vector and scalar potentials; in $\S 2.2$ we derive the general form of the Hamiltonian describing electron motion in an inhomogeneous (dipole) magnetic field and wave fields; in $\S 2.3$ we expand this Hamiltonian around the wave-particle resonance and derive the resonant Hamiltonian describing resonant particle dynamics; in $\S 2.4$ we use this resonant Hamiltonian to derive the main characteristics of resonant particle motion (change of energy due to trapping and due to scattering); $\S 3$ is devoted to the derivation of a generalized kinetic equation describing the evolution of the particle distribution due to nonlinear resonant interactions; in $\S \S 4$ and 5 we apply this equation to describe the evolution of the resonant electron distribution in a system with a parallel propagating whistler mode wave (cyclotron resonance) and in a system with a highly oblique whistler mode wave (Landau resonance); finally, we discuss in $\$ 6$ the main results and possible generalization/modifications of the derived equations.

\section{Main system characteristics}

This section consists of three subsections: $\S 2.1$ describes the distribution of wave electromagnetic field and corresponding vector and scalar potentials, $\S 2.2$ provides a Hamiltonian description of electron motion and $\S 2.3$ outlines the main characteristics of wave-particle resonant interaction.

\subsection{Wave field}

The cold plasma dispersion relation (Stix 1962) provides the relationship between electric and magnetic field components of whistler mode waves. Let $B_{y}=B_{w 0} \operatorname{Re}\left(\mathrm{e}^{\mathrm{i} \phi}\right)=$ $B_{w 0} \cos \phi$ where $\phi$ is the wave phase, then

$$
\left.\begin{array}{c}
\frac{B_{x}}{B_{w 0}}=-\varepsilon_{2} \frac{N^{2} \sin ^{2} \theta-\varepsilon_{3}}{\varepsilon_{3}\left(\varepsilon_{1}-N^{2}\right)} \sin \phi, \quad \frac{B_{z}}{B_{w 0}}=\tan \theta \varepsilon_{2} \frac{N^{2} \sin ^{2} \theta-\varepsilon_{3}}{\varepsilon_{3}\left(\varepsilon_{1}-N^{2}\right)} \sin \phi, \\
\frac{E_{x}}{B_{w 0}}=-\frac{N^{2} \sin ^{2} \theta-\varepsilon_{3}}{\varepsilon_{3} N \cos \theta} \cos \phi, \quad \frac{E_{y}}{B_{w 0}}=\frac{\varepsilon_{2}}{\varepsilon_{1}-N^{2}} \frac{N^{2} \sin ^{2} \theta-\varepsilon_{3}}{\varepsilon_{3} N \cos \theta} \sin \phi, \\
\frac{E_{z}}{B_{w 0}}=-\frac{N \sin \theta}{\varepsilon_{3}} \cos \phi .
\end{array}\right\}
$$

Functions $\varepsilon_{1}, \varepsilon_{2}$ and $\varepsilon_{3}$ are components of the cold plasma dielectric tensor:

$$
\varepsilon_{1}=1-\frac{\Omega_{\mathrm{pe}}^{2}}{\omega^{2}-\Omega_{0}^{2}}, \quad \varepsilon_{2}=\frac{\Omega_{0}}{\omega} \frac{\Omega_{\mathrm{pe}}^{2}}{\omega^{2}-\Omega_{0}^{2}}, \quad \varepsilon_{3}=1-\frac{\Omega_{\mathrm{pe}}^{2}}{\omega^{2}},
$$

where $\omega$ is the wave frequency, $k_{x}$ and $k_{z}$ are two components of the wave vector lying in the $(x, z)$ plane $\left(k_{x}=k \sin \theta, k_{z}=k \cos \theta\right), \Omega_{0}>0$ is the electron gyrofrequency in the background magnetic field directed along the $z$ axis, $\Omega_{\mathrm{pe}}$ the plasma frequency. Wave frequency and wave vector satisfy the wave dispersion relation $N=N(\omega, \theta)$ where $N=k c / \omega$ is the wave refractive index (Stix 1962). Equations (2.1) lead to the 

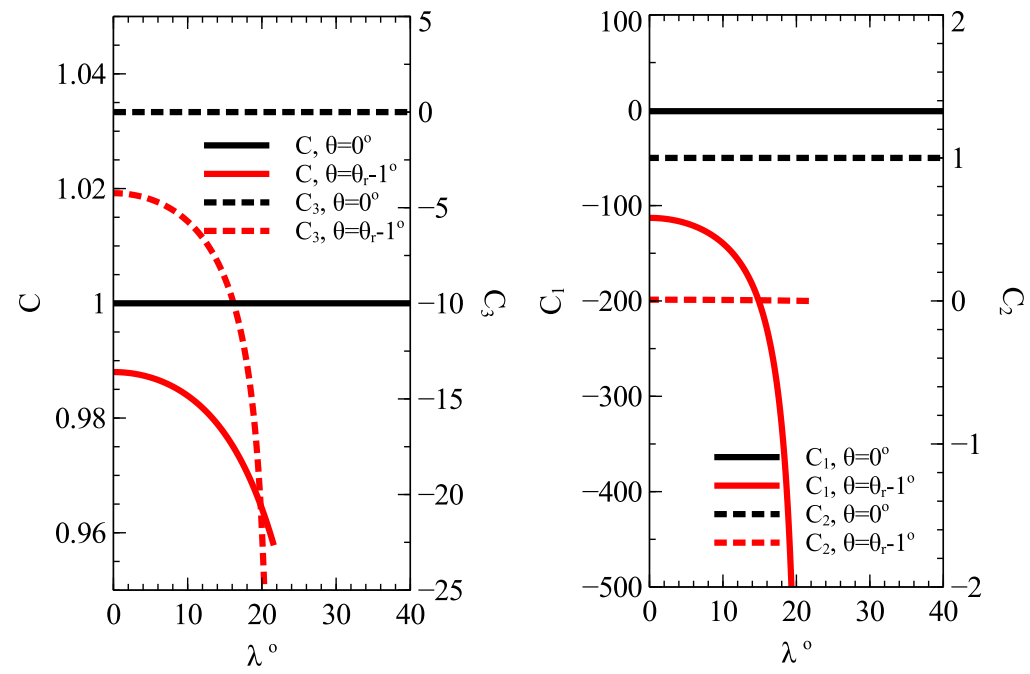

Figure 2. Functions $C_{1}, C_{2}$ given by (2.3), and functions $C=\sqrt{C_{1} C_{2}}, C_{3}=\left(N^{2} \varepsilon_{3}^{-1}-1\right) /$ $N \cos \theta$ for $\omega / \Omega_{0}=0.35$ and $\Omega_{\mathrm{pe}} / \Omega_{0}=4.5$ at $\lambda=0$, considering field-aligned $(\theta=0)$ and highly oblique waves near the resonance cone angle $\theta_{r}$.

following expressions for the total wave magnetic and electric fields:

$$
\left.\begin{array}{c}
B_{w}^{2}=\frac{1}{2 \pi} \int_{0}^{2 \pi}\left(B_{x}^{2}+B_{y}^{2}+B_{z}^{2}\right) \mathrm{d} \phi=\frac{1}{2} B_{w 0}^{2}\left(C_{2} C_{1}+1\right) \\
E_{w}^{2}=\frac{1}{2 \pi} \int_{0}^{2 \pi}\left(E_{x}^{2}+E_{y}^{2}+E_{z}^{2}\right) \mathrm{d} \phi=\frac{1}{2} \frac{B_{w 0}^{2}}{N^{2}}\left(C_{1}\left(1+C_{2}\right)+\left(N^{2} \varepsilon_{3}^{-1} \sin \theta\right)^{2}\right) \\
C_{1}=\left(\frac{N^{2} \varepsilon_{3}^{-1} \sin ^{2} \theta-1}{\cos \theta}\right)^{2}, \quad C_{2}=\left(\frac{\varepsilon_{2}}{\varepsilon_{1}-N^{2}}\right)^{2} .
\end{array}\right\}
$$

For parallel propagating waves $(\theta=0)$, we have $C_{1}=1, N^{2}=\varepsilon_{1}-\varepsilon_{2}, C_{2}=1$ and $B_{w}=B_{w 0}, E_{w}=B_{w 0} / N$. Figure 2 shows the functions $C_{1}, C_{2}$ for different parameters, corresponding notably to parallel and very oblique waves in the radiation belts.

We use the Coulomb gauge to write field components through components of vector potential $\boldsymbol{A}$ and scalar potential $\varphi$ :

$$
\left.\begin{array}{c}
A_{y}=\frac{B_{w 0}}{k} \sqrt{C_{2} C_{1}} \cos \phi, \quad A_{x}=\frac{B_{w 0}}{k} \cos \theta \sin \phi, \\
=-\frac{B_{w 0}}{k} \sin \theta \sin \phi, \quad \varphi=\frac{B_{w 0}}{k N}\left(N^{2} \varepsilon_{3}^{-1}-1\right) \tan \theta \sin \phi .
\end{array}\right\}
$$

We introduce $A_{0}=B_{w 0} / k, C=\sqrt{C_{1} C_{2}}, C_{3}=\left(N^{2} \varepsilon_{3}^{-1}-1\right) / N \cos \theta$ and write $A_{x}=A_{0} \cos \theta \sin \phi, A_{y}=A_{0} C \cos \phi, A_{z}=-A_{0} \sin \theta \sin \phi, \varphi=A_{0} C_{3} \sin \theta \sin \phi$ where $B_{w 0}=B_{w} \sqrt{2 /\left(C^{2}+1\right)}$. Figure 2 shows $C$ and $C_{3} \tan \theta$ as functions of the magnetic latitude $\lambda$ for a dipole model of the background magnetic field $\left(\Omega_{0}(\lambda)=\Omega_{0}(0) \sqrt{1+3 \sin ^{2} \lambda} / \cos ^{6} \lambda\right)$. Highly oblique waves, with $\theta$ close to the resonance cone angle $\theta_{r}$ (Stix 1962), are characterized by a significant variation of vector and scalar potential amplitudes, whereas these amplitudes are almost constant for parallel propagating waves. 


\subsection{Electron dynamics}

We start with the Hamiltonian of an electron (of charge $-e$ and mass $m_{e}$ ) moving in the background magnetic field (described by the $y$-component of the vector potential $A_{b g}=-x B_{0}(z)$ (Bell 1984)) and the wave electromagnetic field (given by (2.4)):

$$
H=\sqrt{m_{e}^{2} c^{4}+c^{2}\left(\boldsymbol{p}+\frac{e}{c} \boldsymbol{A}(x, z, t)+\frac{e}{c} A_{b g}(x, z) \boldsymbol{e}_{y}\right)^{2}}-e \varphi(x, z, t),
$$

where $\boldsymbol{p}$ is the particle generalized momentum. Note that $(x, y, z)$ are Cartesian coordinates, i.e. we do not take into account the magnetic field line curvature of the dipole field (see a more general consideration in Shklyar \& Matsumoto 2009). Although we use a $B_{0}(z)$ function simulating the distribution of a dipole field along magnetic field line, the same consideration is valid for arbitrary ambient magnetic field with a sufficiently large spatial scale of inhomogeneity.

The Hamiltonian (2.5) does not depend on $y$ and the conjugated momentum is constant, $p_{y}=$ const. We consider particles motion around a field line with $x=0$, and thus $p_{y}$ can be taken equal to zero. There are two important assumptions here. First, we assume that the spatial scale of the $B_{0}(z)$ variation is much larger than the wavelength $\sim(\partial \phi / \partial x)^{-1},(\partial \phi / \partial z)^{-1}$. Therefore, the period of particle oscillations in the plane $\left(z, p_{z}\right)$ are much longer than the wave period $\phi$ (wave frequency $-\partial \phi / \partial t$ is approximately the electron gyrofrequency $e B_{0} / m_{e} c$ and much larger than an inverted time scale of $\left(z, p_{z}\right)$ oscillations). Second, we assume a small wave amplitude $e A_{0} / m_{e} c^{2} \ll 1$ (for typical conditions in the Earth radiation belts this limit is satisfied since it means that the wave magnetic field amplitude must be much smaller than 10 nT) and expand the Hamiltonian (2.5) as:

$$
\left.\begin{array}{c}
H=H_{0}+\frac{c e}{H_{0}} \boldsymbol{A}\left(\boldsymbol{p}+\frac{e}{c} A_{b g} \boldsymbol{e}_{y}\right)-e \varphi \\
H_{0}=\sqrt{m_{e}^{2} c^{4}+c^{2} p_{z}^{2}+c^{2} p_{x}^{2}+c^{2}\left(m_{e} x \Omega_{0}(z)\right)^{2}},
\end{array}\right\}
$$

where $\Omega_{0}=e B_{0}(z) / m_{e} c$. Considering the unperturbed system $H=H_{0}$, we introduce the adiabatic invariant (magnetic moment):

$$
I_{x}=\frac{1}{2 \pi} \oint p_{x} \mathrm{~d} x=\frac{H_{0}^{2}-m_{e}^{2} c^{4}-c^{2} p_{z}^{2}}{2 m_{e} c^{2} \Omega_{0}} .
$$

The corresponding coordinates $\left(x, p_{x}\right)$ can be rewritten as: $p_{x}=-\sqrt{2 I_{x} \Omega_{0} m_{e}} \sin \psi$ and $x=\sqrt{2 I_{x} / \Omega_{0} m_{e}} \cos \psi$ where $I_{x}, \psi$ are conjugated variables (i.e. the transformation $\left(x, p_{x}\right)$ to $\left(\psi, I_{x}\right)$ is canonical (Landau \& Lifshitz 1988)). To introduce these variables we use the generating function $G_{0}=\int p_{x} \mathrm{~d} x+p_{z \text {,new }} z$ where

$$
p_{z, \text { new }}=p_{z}-\frac{\partial G_{0}}{\partial z}=p_{z}-I_{x} \frac{1}{2} \frac{\partial \ln \Omega_{0}}{\partial z} \sin 2 \psi
$$

and $z$ does not change. Therefore, the Hamiltonian (2.6) can be rewritten in new variables:

$$
\left.\begin{array}{c}
H=m_{e} c^{2} \sqrt{1+\frac{1}{m_{e}^{2} c^{2}}\left(p_{z, \text { new }}+I_{x} \frac{1}{2} \frac{\partial \ln \Omega_{0}}{\partial z} \sin 2 \psi\right)^{2}+\frac{\rho^{2} \Omega_{0}^{2}}{c^{2}}}+H_{1}, \\
H_{1}=\frac{1}{\gamma_{0}}\left(\frac{\rho \Omega_{0}}{c}\left(e A_{y} \cos \psi-e A_{x} \sin \psi\right)+\frac{e A_{z}}{m_{e} c}\left(p_{z, \text { new }}+I_{x} \frac{1}{2} \frac{\partial \ln \Omega_{0}}{\partial z} \sin 2 \psi\right)\right)-e \varphi,
\end{array}\right\}
$$


where $\rho=\sqrt{2 I_{x} / m_{e} \Omega_{0}}$. There is a fast oscillating term $\sim \sin 2 \psi$. This term results in variation of adiabatic invariant $I_{x}\left(\dot{I}_{x}=-\partial H / \partial \psi\right)$. The amplitude of this term is $\sim \partial \ln \Omega_{0} / \partial z$, i.e. $I_{x}$ is conserved with accuracy $\sim \partial \ln \Omega_{0} / \partial z$. In the absence of wave perturbations (i.e. in the absence of external forces depending on the wave phase $\phi$ ) we can always introduce the improved adiabatic invariant $J_{x}$, which is conserved with accuracy $\sim\left(\partial \ln \Omega_{0} / \partial z\right)^{2}$ (Arnold Kozlov \& Neishtadt 2006). In a system with the wave depending on the phase $\phi$, the variation of $I_{x}$ is determined by the resonance of the wave phase $n \psi+\phi, n=0, \pm 1, \pm 2, \ldots$ (see below). For resonant particles with $\mathrm{d}(n \psi+\phi) / \mathrm{d} t \approx 0$ the phase $2 \psi$ is a fast oscillating non-resonant phase, and, after expansion of $\gamma_{\text {new }}$ over $\partial \ln \Omega_{0} / \partial z$, the corresponding term $\sim \sin 2 \psi$ can be omitted as a fast oscillating term with mean zero value. Therefore, we can rewrite the Hamiltonian (2.9) as

$$
\left.\begin{array}{c}
H=m_{e} c^{2} \gamma+H_{1}, \gamma=\sqrt{1+\frac{p_{z}^{2}}{m_{e}^{2} c^{2}}+\frac{\rho^{2} \Omega_{0}^{2}}{c^{2}}} \\
=\frac{1}{\gamma}\left(\frac{\rho \Omega_{0}}{c}\left(e A_{y} \cos \psi-e A_{x} \sin \psi\right)+\frac{e A_{z}}{m_{e} c} p_{z}\right)-e \varphi,
\end{array}\right\}
$$

were $p_{z \text {,new }} \approx p_{z}$.

We then substitute equations (2.4) into the $H_{1}$ expression to get:

$$
H_{1}=\frac{e A_{0}}{\gamma} \frac{\rho \Omega_{0}}{c}(C \cos \phi \cos \psi-\cos \theta \sin \phi \sin \psi)-e A_{0}\left(\frac{p_{z}}{\gamma m_{e} c}+C_{3}\right) \sin \theta \sin \phi,
$$

where wave phase $\phi$ can be written as

$$
\phi=\phi_{\|}+k_{x} \rho \cos \psi, \quad \phi_{\|}=\int^{z} k_{z}(\tilde{z}) \mathrm{d} \tilde{z}-\omega t .
$$

Using the Jacobi-Anger expansion, we rewrite (2.11) as

$$
\left.\begin{array}{rl}
H_{1}= & e A_{0} \sum_{n=-\infty}^{\infty} h^{(n)}\left(I_{x}, p_{z}, z\right) \sin \left(\phi_{\|}+n \frac{\pi}{2}-n \psi\right) \\
h^{(n)}= & \frac{\rho \Omega_{0}}{2 c \gamma}\left((C-\cos \theta) J_{n-1}\left(k_{x} \rho\right)-(C+\cos \theta) J_{n+1}\left(k_{x} \rho\right)\right) \\
& -\left(\frac{p_{z}}{\gamma m_{e} c}+C_{3}\right) \sin \theta J_{n}\left(k_{x} \rho\right),
\end{array}\right\}
$$

where $J_{n}$ is the Bessel function. We consider waves with insufficiently strong amplitudes to allow an overlap of resonances, i.e. such that each term in the sum of resonances can be considered independently (i.e. the resonance width $\sim \sqrt{\left|e A_{0} h^{(n)} / m_{e}\right|}$ is much smaller than the smallest distance between two resonances $\sim \Omega_{0} / k \cos \theta$, see the corresponding discussion in Shklyar 1981). For one particular $n$, equation (2.10) takes the form

$$
H^{(n)}=m_{e} c^{2} \gamma+e A_{0} h^{(n)} \sin \left(\phi_{\|}+n \frac{\pi}{2}-n \psi\right) .
$$

Numerically solving the Hamiltonian equations for the $\left(z, p_{z}\right),\left(\psi, I_{x}\right)$ variables for the Hamiltonian (2.14) allows us to describe the resonant interaction of charged particles with a whistler mode wave. In the next subsection, we investigate the Hamiltonian (2.14) to determine the main characteristics of this resonant interaction. 


\subsection{Wave-particle resonance}

First, we would like to consider a conservative system with conserved energy, whereas the Hamiltonian (2.14) depends on time through phase $\phi_{\|}$. To exclude this temporal dependence, we introduce the new conjugated variables $\left(\zeta_{n}, I\right)$ and $\left(z, P_{z}\right)$ with $\zeta_{n}=$ $\phi_{\|}-n \psi+n \pi / 2$. This variable change excludes phase $\psi$ from the Hamiltonian and, thus, the new momentum $\tilde{I}_{x}$ is constant - i.e. instead of two pairs of conjugated variables $\left(z, p_{z}\right),\left(\psi, I_{x}\right)$ we introduce $\left(z, P_{z}\right),\left(\zeta_{n}, I\right)$. To this aim, we use the generating function $G_{1}=\left(\phi_{\|}+n \psi+n \pi / 2\right) I+z P_{z}+\psi \tilde{I}_{x}$ corresponding to:

$$
\zeta_{n}=\phi_{\|}+n \frac{\pi}{2}-n \psi, \quad p_{z}=k_{z} I+P_{z}, \quad I_{x}=-n I+\tilde{I}_{x} .
$$

Therefore, the new Hamiltonian $F=H^{(n)}+\partial G_{1} / \partial t$ takes the form

$$
\left.\begin{array}{c}
F=-\omega I+m_{e} c^{2} \gamma+e A_{0} h^{(n)} \sin \zeta_{n} \\
\gamma=\sqrt{1+\frac{\left(k_{z} I+P_{z}\right)^{2}}{m_{e}^{2} c^{2}}+\frac{2 \Omega_{0}}{m_{e} c^{2}}\left(\tilde{I}_{x}-n I\right),}
\end{array}\right\}
$$

where $h^{(n)}=h^{(n)}\left(\tilde{I}_{x}-n I, k_{z} I+P_{z}, z\right)$. The resonance condition $\dot{\zeta}_{n}=0$ can be written through Hamiltonian equations:

$$
\dot{\zeta}_{n}=\frac{\partial F}{\partial I}=-\omega+\frac{k_{z}\left(k_{z} I+P_{z}\right)-m_{e} \Omega_{0} n}{m_{e} \gamma}=0,
$$

where we omit perturbations $\sim e A_{0} \ll m_{e} c^{2}$. The solution of (2.17) provides the resonant value $I_{R}$ of momentum $I$ :

$$
\frac{k_{z} I_{R}}{m_{e} c}=-\frac{P_{z}}{m_{e} c}-\frac{\omega_{n}}{N_{\|}}+\frac{1}{\sqrt{N_{\|}^{2}-1}} \sqrt{1+\beta_{\perp}^{2}-\frac{\omega_{n}^{2}}{N_{\|}^{2}}-2 \frac{\omega_{n}}{N_{\|}} \frac{P_{z}}{m_{e} c}}
$$

where $N_{\|}=k_{z} c / \omega, \omega_{n}=-n \Omega_{0} / \omega$, and $\beta_{\perp}=\sqrt{2 \tilde{I}_{x} \Omega_{0} / m_{e} c^{2}}$. Particles with $I=I_{R}$ are in resonance with the wave. Therefore, to consider such resonant particles we expand the Hamiltonian (2.4) around $I_{R}$ :

$$
\begin{gathered}
F=\Lambda+\frac{1}{2} m_{e} c^{2} g\left(I-I_{R}\right)^{2}+e A_{0} h_{R}^{(n)} \sin \zeta_{n} \\
\Lambda=-\omega I_{R}+m_{e} c^{2} \gamma_{R}=\frac{m_{e} c^{2}}{N_{\|}^{2}}\left(\frac{N_{\|} P_{z}}{m_{e} c}+\omega_{n}+\left(N_{\|}^{2}-1\right) \gamma_{R}\right) \\
\gamma_{R}=\frac{N_{\|}}{\sqrt{N_{\|}^{2}-1}} \sqrt{1+\beta_{\perp}^{2}-\frac{\omega_{n}^{2}}{N_{\|}^{2}}-2 \frac{\omega_{n}}{N_{\|}} \frac{P_{z}}{m_{e} c}} \\
g=\left.\frac{\partial^{2} \gamma}{\partial I^{2}}\right|_{I=I_{R}}=\left(N_{\|}^{2}-1\right) \frac{\omega^{2}}{m_{e}^{2} c^{4} \gamma_{R}},
\end{gathered}
$$

where $h_{R}^{(h)}=h^{(n)}\left(n I_{R}+\tilde{I}_{x}, k_{z} I_{R}+P_{z}, z\right)$. We introduce variables $K=I-I_{R}, p, s$ through the generating function $G_{2}=\left(I-I_{R}\right) \zeta_{n}+P_{z} s$ (we keep the old notation for $\zeta_{n}$, because this transformation does not change the phase):

$$
K=I-I_{R}, \quad p=P_{z}-\frac{\partial I_{R}}{\partial z} \zeta_{n}, \quad s=z+\frac{\partial I_{R}}{\partial P_{z}} \zeta_{n} .
$$


The new Hamiltonian $W=\Lambda(s, p)+m_{e} c^{2} g(s, p) K^{2} / 2+e A_{0} h_{R}^{(n)}(s, p) \sin \zeta_{n}$ contains the $\Lambda$ function depending on $(s, p)$. Terms $\sim \partial I_{R} / \partial z, \partial I_{R} / \partial P_{z}$ in (2.20) are much smaller than $\left(z, P_{z}\right)$ terms due to the condition $\partial / \partial z \ll k_{z}$. Therefore, we can expand the function $\Lambda$ :

$$
\Lambda\left(z, P_{z}\right)=\Lambda\left(s-\frac{\partial I_{R}}{\partial P_{z}} \zeta_{n}, p+\frac{\partial I_{R}}{\partial z} \zeta_{n}\right)=\Lambda(s, p)+\left(\frac{\partial I_{R}}{\partial P_{z}} \frac{\partial \Lambda}{\partial z}-\frac{\partial I_{R}}{\partial z} \frac{\partial \Lambda}{\partial P_{z}}\right) \zeta_{n}=\Lambda-r \zeta_{n}
$$

where $r=\left\{\Lambda, I_{R}\right\}_{z, P_{z}} \approx\left\{\Lambda, I_{R}\right\}_{s, p}$. Therefore, the new Hamiltonian $W$ can be split into two Hamiltonians $W=W_{\text {slow }}+\mathfrak{F}$, where $W_{\text {slow }}=\Lambda(s, p) \sim m_{e} c^{2}$ describes the slow evolution of the $(s, p)$ variables, while $\mathfrak{F} \sim e A_{0} \ll m_{e} c^{2}$ depends on these variables as on the parameters and describes the fast variations of $\left(\zeta_{n}, K\right)$ :

$$
\left.\begin{array}{c}
\mathfrak{F}=\frac{1}{2} \frac{N_{\|}^{2}-1}{\gamma_{R}} \frac{\omega^{2}}{m_{e}^{2} c^{4}} K^{2}-r \zeta_{n}+e A_{0} h_{R}^{(n)} \sin \zeta_{n} \\
m_{e} c^{2} D N_{\|}^{2} \frac{1}{N_{\|}^{2}-1}\left(\frac{\left(\gamma_{R}-\omega_{n}\right)^{2}}{N_{\|}^{2}} \frac{\partial \ln N_{\|}}{\partial \ln \Omega_{0}}+\frac{\omega_{n}}{N_{\|}} \frac{p}{m_{e} c}-\frac{1}{2} \beta_{\perp}^{2}\right),
\end{array}\right\}
$$

where $D=c\left(\partial \ln \Omega_{0} / \partial s\right) / N_{\|} \omega \ll 1$ is a dimensionless factor determining the scale ratio of the inhomogeneity scale $\sim(\partial / \partial z)^{-1}$ and wavelength scale $\sim 1 / k_{z}$.

\subsection{Characteristics of resonant interaction}

Using the resonant Hamiltonian (2.22), we can derive the main characteristics of the corresponding resonant wave-particle interaction. We start with the determination of values $(s, p)$ where the particle reaches resonance with the wave. Each particle trajectory is characterized by two constants: the generalized energy $\gamma-\omega I / m_{e} c^{2}=$ const. (i.e. the normalized value of $F$ from (2.16) omitting small terms) and $\tilde{I}_{x}$. Momenta $\tilde{I}_{x}$ and $I$ are linearly related to each other $\left(\tilde{I}_{x}=n I+I_{x}\right)$ and we define constant $\tilde{I}_{x}$ to be equal to the initial $I_{x}$. Thus, the initial value of $I$ equals zero and $\gamma_{\text {init }}=-\omega I_{R} / m_{e} c^{2}+\gamma_{R}$, where $\gamma_{\text {init }}$ is the initial value of the energy. We substitute this equation into (2.18) and solve the obtained equation relative to resonant momentum $p_{R} \approx P_{z}$ :

$$
\frac{p_{R}}{m_{e} c}=N_{\|} \gamma_{\text {init }}-N_{\|} \omega_{n} \pm \sqrt{N_{\|}^{2}-1} \sqrt{1+\varepsilon+\omega_{n}^{2}},
$$

where we introduce a new function $\varepsilon=\beta_{\perp}^{2}-2 \gamma_{\text {init }} \omega_{n}$. For every $\gamma_{\text {init }}$, equation (2.23) defines the resonant curve $p_{R}=p_{R}(s)$. The particles trapped into the resonance move along such curves, and thus particle energy $\gamma=\gamma_{R}$ and all coefficients of (2.22) can be rewritten as functions of $s$ only (i.e. $\omega_{n}(s), N_{\|}(s)$ ):

$$
\left.\begin{array}{c}
\gamma=\left|\omega_{n}-\frac{N_{\|}}{\sqrt{N_{\|}^{2}-1}} \sqrt{1+\varepsilon+\omega_{n}^{2}}\right| \\
\frac{p_{R}}{m_{e} c}=N_{\|} \gamma_{\text {init }}-N_{\|} \omega_{n} \pm \sqrt{N_{\|}^{2}-1} \sqrt{1+\varepsilon+\omega_{n}^{2}} \\
\left.r=\frac{1}{2} \frac{m_{e} c^{2} D N_{\|}^{2}}{N_{\|}^{2}-1} \frac{1}{\gamma}\left(1-\gamma^{2}+2 \frac{\left(\gamma-\omega_{n}\right)^{2}}{N_{\|}^{2}} \frac{\partial \ln N_{\|}}{\partial \ln \Omega_{0}}+\frac{\gamma^{2}-\omega_{n}^{2}}{N_{\|}^{2}}\right) \cdot\right\}
\end{array}\right\}
$$


The resonant coordinate $s_{R}(\gamma)$ is defined from the first equation of (2.24). Substituting $s_{R}(\gamma)$ to $(2.22)$ and fixing $\tilde{I}_{x}$, we obtain $r=r(\gamma)$.

Defining resonant $p_{R}=p_{R}\left(\gamma, s_{R}\right)$ and $s_{R}=s_{R}(\gamma)$ (see (2.23), (2.24)), we can provide a quantitative description of the particle resonant interaction. Let us start with the time scale between two successive resonance crossings, $T$. We assume that there is one resonant interaction per period of slow $\left(z, p_{z}\right)$ motion, i.e. the wave propagates from $z=0$ to positive $z$ and there is no wave at negative $z$. This assumption also means that waves generated at $z=0$ and propagating to high $z$ are not reflected at high $z$ and do not return back to $z=0$. Spacecraft observations (e.g. Agapitov et al. 2011) and chorus wave modelling including the Landau damping effect (e.g. Bortnik et al. 2006; Breuillard et al. 2013; Chen et al. 2013) suggest that in the Earth's radiation belt, strong damping at high $z$ and spreading of wave power result in a very small intensity of the waves propagating back from high $z$ toward the $z=0$ plane. Thus, $T$ can be calculated from the unperturbed Hamiltonian (2.14):

$$
T\left(\gamma, \tilde{I}_{x}\right)=\oint \frac{\mathrm{d} z}{\partial H / \partial p_{z}}=\frac{\gamma}{\sqrt{\gamma^{2}-1}} \oint\left(1-\frac{\varepsilon+2 \omega_{n} \gamma}{\gamma^{2}-1}\right)^{-1 / 2} \frac{\mathrm{d} z}{c},
$$

where we rewrite $I_{x}=-n I+\tilde{I}_{x}$ through $\tilde{I}_{x}=$ const., $-\omega I / m_{e} c^{2}+\gamma=\gamma_{\text {init }}$, and $\varepsilon=\Omega_{0} \tilde{I}_{x} / m_{e} c^{2}-2 \gamma_{\text {init }} \omega_{n}$. The period (2.25) depends only on the energy $\gamma$. The assumption that there is a single resonance during one bounce period does not follow from the system properties; it is used to simplify the derived equations. In more realistic systems, charged particles can resonate with the same wave several times along the bounce trajectory, and such multi-resonances can significantly influence charged particle motion (e.g. Shklyar 1981). To include the effects of several resonances within one bounce period, we would need to describe the time scale between two successive resonances depending on the wave characteristics. This generalization of the derived equations will be considered in a future study.

Over one period $T$, particle scattering results in a change of momentum $I$, and a corresponding change of energy $\gamma: \omega \Delta I / m_{e} c^{2}=\Delta \gamma$ : this change can be written as

$$
\left.\begin{array}{rl}
\Delta \gamma & =\frac{\omega \Delta I}{m_{e} c^{2}}=-\frac{\omega}{m_{e} c^{2}} \int \frac{\partial F}{\partial \zeta_{n}} \mathrm{~d} t=-\frac{2 e A_{0} h_{R}^{(n)} \omega}{m_{e} c^{2}} \int_{-\infty}^{\zeta_{n}^{*}} \frac{\cos \zeta_{n} \mathrm{~d} \zeta_{n}}{\dot{\zeta}_{n}} \\
& =\sqrt{\frac{2 e A_{0} \gamma_{R} h_{R}^{(n)}}{m_{e} c^{2}\left(N_{\|}^{2}-1\right)}} f\left(\zeta_{n}^{*}, a\right) \\
f\left(\zeta_{n}^{*}, a\right) & =\int_{-\infty}^{\zeta_{n}^{*}} \frac{\sqrt{a} \cos \zeta_{n} \mathrm{~d} \zeta_{n}}{\sqrt{\zeta_{n}-\zeta_{n}^{*}+a\left(\sin \zeta_{n}^{*}-\sin \zeta_{n}\right)}},
\end{array}\right\}
$$

where the integration is performed along the trajectory of particles approaching the resonance from infinity, interacting with waves with the resonance value of phase $\zeta_{n}^{*}$ and escaping from the resonance toward infinity. Note that integral (2.26) is written for negative $r\left(\zeta_{n}\right.$ changes from $-\infty$ to $\left.\zeta_{n}^{*}\right)$, whereas for positive $r$ the integration should be performed for $\zeta_{n} \in\left[\zeta_{n}^{*},-\infty\right]$. The parameter $a=e A_{0} h_{R}^{(n)} / r$ and other parameters should be evaluated at $\left(p_{R}, s_{R}\right)$, i.e. all these parameters depend only on $\gamma$. We further introduce the new variable $\xi$ through the particle energy evaluated at 
$\zeta_{n}^{*}: 2 \pi \xi=a \sin \zeta_{n}^{*}-\zeta_{n}^{*}$. The function $f\left(\zeta_{n}^{*}, a\right)=f(\xi, a)$ is a periodic function of $\xi$, whereas $\xi$ depends on the initial value of $\zeta_{n}$ and on the initial particle gyrophase (see, e.g. Karpman, Istomin \& Shklyar 1975). We do not include any information about particle gyrophase in our present description. Thus, we calculate here the average value of $f$ over $\xi$. The distribution of $\xi$ is assumed to be uniform $\xi \in[0,1]$ (see corresponding numerical tests in Itin, Neishtadt \& Vasiliev 2000). An interesting and crucial property of the function $f(\xi, a)$ is that $\langle\Delta I\rangle_{\xi}=-S_{\text {sep }} / 2 \pi$ (Karpman et al. 1975; Neishtadt 1999; Dolgopyat 2012) where $S_{\text {sep }}$ is defined as:

$$
S_{\text {sep }}=\oint K \mathrm{~d} \zeta_{n}=2^{3 / 2} \sqrt{\frac{\gamma_{R} r}{N_{\|}^{2}-1} \frac{m_{e}^{2} c^{4}}{\omega^{2}}} \int_{\zeta_{\min }}^{\zeta_{\max }} \sqrt{\zeta_{n}-\zeta_{\max }+a\left(\sin \zeta_{\max }-\sin \zeta_{n}\right)} \mathrm{d} \zeta_{n}
$$

and $\zeta_{\min }$ is defined by equation $a \cos \zeta_{\min }=1$ and $\zeta_{\max }$ is defined by equation $\zeta_{\min }-$ $a \sin \zeta_{\min }=\zeta_{\max }-a \sin \zeta_{\max }$. Therefore, we can introduce

$$
\left.\begin{array}{c}
\langle\Delta \gamma\rangle=\sqrt{\frac{2 e A_{0} \gamma_{R} h_{R}^{(n)}}{m_{e} c^{2}\left(N_{\|}^{2}-1\right)}} f_{S}(a) \\
f_{S}(a)=\frac{1}{\pi \sqrt{a}} \int_{\zeta_{\min }}^{\zeta_{\max }} \sqrt{\zeta_{n}-\zeta_{\max }+a\left(\sin \zeta_{\max }-\sin \zeta_{n}\right)} \mathrm{d} \zeta_{n} .
\end{array}\right\}
$$

For $|a|<1$ we have $f_{S}=0$, and for $|a| \gg 1$ we have $f_{S}=\sqrt{2} / \pi$. The change $\langle\Delta \gamma\rangle$ depends only on $\gamma$.

In addition to scattering, one must also consider particle trapping (e.g. Omura et al. 2007; Shklyar \& Matsumoto 2009; Albert Tao \& Bortnik 2013). A particle trapped by a wave starts moving with the resonant velocity (i.e. with a resonant momentum given by (2.23)). During such motion, the particle's $\gamma$ varies like $\gamma_{R}$, i.e. the particle remains a long time in resonance with the wave. Trapped particles fill an area in the $\left(\zeta_{n}, K\right)$ plane equal to $S_{\text {res }}$, i.e. the phase volume of the trapped particles is given by the same expression as the one determining the energy change of the scattered particles (e.g. Karpman et al. 1975; Shklyar 2011). Trapping of new particles into this volume requires that the area $S_{\text {sep }}$ evaluated along the trapped trajectory grows with time: $\mathrm{d} S_{\text {sep }} / \mathrm{d} t=\left\{S_{\text {sep }}, \Lambda\right\}_{(s, p)}>0$. This condition is satisfied either for the wave amplitude $e A_{0} h^{(n)}$ growing along the resonant trajectory or for a decreasing $|r|$ (e.g. a reduction of the inhomogeneity factor $D$, see (2.22)). The condition $\mathrm{d} S_{\text {sep }} / \mathrm{d} t>0$ determines a certain range of $\gamma$ values. Trapped particles escape from the resonance when $\mathrm{d} S_{\text {sep }} / \mathrm{d} t<0$ and $\gamma=\gamma^{*}$, where $\gamma^{*}$ is a solution of equation $S_{\text {sep }}\left(\gamma^{*}\right)=S_{\text {sep }}\left(\gamma_{\text {trap }}\right)$, where $\gamma_{\text {trap }}$ is the $\gamma$ value at the moment of trapping (e.g. Neishtadt, Petrovichev \& Chernikov 1989; Itin et al. 2000; Vasiliev et al. 2011). We consider $S_{\text {sep }}$ dependence on $\gamma$ and define two ranges of $\gamma$ (see scheme in figure 3):

$$
\frac{\mathrm{d} S_{\text {sep }}}{\mathrm{d} t}<0 \text { for } \gamma \in \mathrm{Z}_{\mathrm{esc}}, \quad \text { and } \quad \frac{\mathrm{d} S_{\text {sep }}}{\mathrm{d} t}>0 \text { for } \gamma \in \mathrm{Z}_{\text {trap }} \text {. }
$$

Therefore, trapping transports particles toward a position in $Z_{\text {esc }}$ from a position in $\mathrm{Z}_{\text {trap }}$. In this study, we assume that wave intensity maximizes at intermediate $z$ and drops to zero at both $z=0$ and large $z$. Therefore, trapping into resonance and escape from the resonance occur within the same hemisphere $(z>0)$. We do not consider the more complicated situation where waves with trapped particles can cross the $z=0$ plane several times. Therefore, particle transport in energy space is limited by the time 


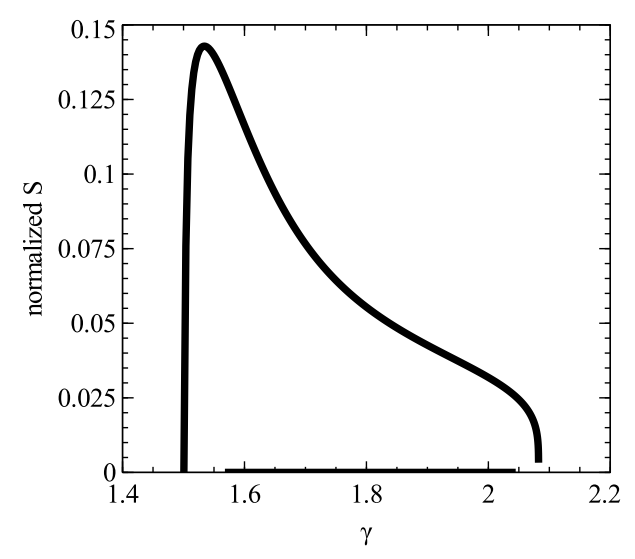

FIGURE 3. Normalized area $S_{\text {sep }}(\gamma)$ given by (2.27).

scale of trapped particle motion in the resonance (approximately a quarter of particle bounce period). This transport is described by the map $\gamma=Y\left(\gamma^{*}\right)$ where $\gamma \in \mathrm{Z}_{\mathrm{esc}}$ and $\gamma^{*} \in \mathrm{Z}_{\text {trap }}$. The function $Y\left(\gamma^{*}\right)$ is defined by equation $S_{\text {sep }}\left(\gamma^{*}\right)=S_{\text {sep }}(\gamma)$. As shown in Artemyev et al. (2016), to write the generalized Fokker-Plank equation, we need to determine only the relation $\langle\Delta \gamma\rangle=\langle\Delta \gamma\rangle(\gamma)$ and the map $\gamma=Y\left(\gamma^{*}\right)$.

\section{Generalized Fokker-Planck equation}

Resonant wave-particle interactions lead to particle scattering, which can be defined via two physical quantities: a diffusion coefficient $D_{\gamma \gamma}=\left\langle(\Delta \gamma)^{2}\right\rangle / 2 T(\gamma)$ and a drift velocity $V_{\gamma}=\langle\Delta \gamma\rangle / T(\gamma)$. (Note, we consider unidirectional wave propagation, and thus there is a finite $\Delta \gamma$.) These two characteristics describe respectively particle diffusion and particle drift in $\gamma$ space (for fixed $\tilde{I}_{x}=$ const.). The corresponding evolution of the particle distribution function can be written in terms of two operators

$$
\left.\begin{array}{c}
\hat{L}_{D} \Psi=\frac{\partial}{\partial \gamma}\left(D_{\gamma \gamma} \frac{\partial \Psi}{\partial \gamma}\right) \\
\hat{L}_{V} \Psi=-\frac{\partial V_{\gamma} \Psi}{\partial \gamma}
\end{array}\right\}
$$

The third effect is particle trapping and fast transport. This effect can be described by probabilistic operators (Solovev \& Shklyar 1986; Artemyev et al. 2014; Omura et al. 2015). This kind of operator depends on the probability of trapping $\Pi=-\mathrm{d}\langle\Delta I\rangle / \mathrm{d} I$ (Artemyev et al. 2016, 2017a) and can be written as

$$
\left.\begin{array}{c}
\hat{L}_{T} \Psi=-\frac{\Pi}{T} \Psi=\frac{\Psi}{T} \frac{\mathrm{d}\langle\Delta \gamma\rangle}{\mathrm{d} \gamma}, \quad \gamma \in \mathrm{Z}_{\text {trap }} \\
\Psi=-\frac{\Pi^{*}}{T^{*}} \Psi^{*}\left|\frac{\mathrm{d} \gamma^{*}}{\mathrm{~d} \gamma}\right|=\frac{\Psi^{*}}{T^{*}} \frac{\mathrm{d}\langle\Delta \gamma\rangle}{\mathrm{d} \gamma}, \quad \gamma \in \mathrm{Z}_{\mathrm{esc}},
\end{array}\right\}
$$

where $\Pi^{*}=\Pi\left(\gamma^{*}\right), \Psi^{*}=\Psi(\gamma)$ and $\gamma^{*}$ is a solution of equation $\gamma=Y\left(\gamma^{*}\right)$. Note that in (3.2) we use the linear relation between $I$ and $\gamma$. Combining equations (3.1) 
and (3.2), and using $\langle\Delta \gamma\rangle=T(\gamma) V_{\gamma}$, we obtain the final form of the equation for $\Psi$ $\left(\partial \Psi / \partial t=\hat{L}_{D} \Psi+\hat{L}_{V} \Psi+\hat{L}_{T} \Psi\right)$ :

$$
\left.\begin{array}{rl}
\frac{\partial \Psi}{\partial t}= & -V_{\gamma} \frac{\partial \Psi}{\partial \gamma}+\frac{1}{T} \frac{\mathrm{d} T}{\mathrm{~d} \gamma} V \Psi+\frac{\partial}{\partial \gamma}\left(D_{\gamma \gamma} \frac{\partial \Psi}{\partial \gamma}\right), \quad \gamma \in \mathrm{Z}_{\text {trap }} \\
\frac{\partial \Psi}{\partial t}= & -V_{\gamma} \frac{\partial \Psi}{\partial \gamma}-\frac{\partial V_{\gamma}}{\partial \gamma}\left(\Psi-\frac{T}{T^{*}} \Psi^{*}\right)+\frac{1}{T^{*}} \frac{\mathrm{d} T^{*}}{\mathrm{~d} \gamma} V_{\gamma} \Psi^{*} \\
& +\frac{\partial}{\partial \gamma}\left(D_{\gamma \gamma} \frac{\partial \Psi}{\partial \gamma}\right), \quad \gamma \in \mathrm{Z}_{\mathrm{esc}} .
\end{array}\right\}
$$

We introduce the action of the averaged system $J$ as $\mathrm{d} J / \mathrm{d} \gamma=T(\gamma) / 2 \pi$ and rewrite (3.3) for $J$ using the function $\Psi_{J}=2 \pi \Psi / T, V_{J}=V_{\gamma} T / 2 \pi, D_{J J}=D_{\gamma \gamma} T^{2} / 4 \pi^{2}$ (Artemyev et al. 2017a):

$$
\frac{\partial \Psi_{J}}{\partial t}=-V_{J} \frac{\partial \Psi_{J}}{\partial J}+\frac{\partial}{\partial J}\left(D_{J J} \frac{\partial \Psi_{\mathrm{J}}}{\partial J}\right)-\frac{\partial V_{J}}{\partial J}\left(\Psi_{J}-\Psi_{J}^{*}\right) \Theta+\frac{\partial}{\partial J}\left(\delta V_{J} \Psi_{J}\right)+C_{J},
$$

where $\delta V_{J}=D_{J J} \partial \ln T / \partial J$, the function $\Theta$ is equal to one when $\gamma \in \mathrm{Z}_{\text {esc }}$ (or $\mathrm{d} V_{J} / \mathrm{d} J>$ 0 ) and $\Theta$ is zero when $\gamma \in \mathrm{Z}_{\text {trap }}$ (or $\mathrm{d} V_{J} / \mathrm{d} J<0$ ); see (2.29)). An additive factor $C_{J}$ includes small terms proportional to $\sim \partial \ln T / \partial J$ and we omit this factor for further calculations. There are two drift terms in (3.4): $\sim V_{J}$ and $\sim \delta V_{J} \sim D_{J J}$. Equation (2.26) shows that $V_{J} \sim \Delta \gamma \sim \sqrt{e A_{0} / m_{e} c^{2}}$, whereas $\delta V_{J} \sim(\Delta \gamma)^{2} \sim e A_{0} / m_{e} c^{2} \ll V_{J}$. Thus, we keep only one drift term $\sim V_{J}$ in (3.4). Moreover, we should note that to write (3.1), we omit terms smaller than $\sim O\left(e A_{0} / m_{e} c\right)$. Equation (3.4) describes the evolution of the $\Psi_{J}(J)$ distribution, which can be transformed back to the $\Psi(\gamma)$ distribution using $\mathrm{d} J / \mathrm{d} \gamma=T(\gamma) / 2 \pi$. This evolution includes many resonant interactions (many trappings and scatterings), but each interaction is localized in time (trapping lasts for a time interval smaller than the bounce period). Thus, equation (3.4) describes the long-term evolution of the particle distribution function averaged over a bounce period.

\section{Parallel propagating waves: cyclotron resonance}

In this section, we consider nonlinear resonant electron interaction with parallel propagating whistler mode waves (i.e. with $k_{x}=0$ and $C_{1}=C_{2}=C=1$ in (2.13)). Therefore, $n=-1$ and the Hamiltonian $H_{1}$ takes the form

$$
H_{1}=-e A_{0} \frac{\rho \Omega_{0}}{c \gamma} \sin \left(\phi_{\|}+\psi-\pi / 2\right)
$$

and there are no other resonances (the corresponding Bessel functions in (2.13) are equal to zero). We consider a magnetic field model with $\Omega_{0}(s / R)$, where $R$ is the spatial scale of the field gradient. We also use the dipole magnetic latitude $\lambda$ instead of $s: \mathrm{d} s / \mathrm{d} \lambda=R \sqrt{1+3 \sin ^{2} \lambda} \cos \lambda$ and $\Omega_{0}=\Omega_{0}(0) \sqrt{1+3 \sin ^{2} \lambda} / \cos ^{6} \lambda$. Introducing the dimensionless frequency $\varpi=\omega_{-1}=\Omega_{0}(s) / \omega$ and using the simplified dispersion relation valid for parallel propagating waves (Stix 1962), we write:

$$
N_{\|}=\frac{\Omega_{\mathrm{pe}}}{\omega}(\varpi-1)^{-1 / 2}, \quad \frac{\partial \ln N_{\|}}{\partial \ln \Omega_{0}}=-\frac{1}{2} \varpi(\varpi-1)^{-1},
$$


where $\Omega_{\text {pe }}=$ const. is the plasma frequency. Note that $N_{\|}$is the refractive index $N$ of parallel propagating waves. Using $\omega_{n}=-n \Omega_{0} / \omega=\varpi$, we rewrite $\varepsilon=\left(\beta_{\perp}^{2}-2 \gamma_{\text {init }} \omega_{n}\right)$ as:

$$
\varepsilon=\beta_{\perp}^{2}-2 \gamma_{\text {init }} \omega_{n}=\frac{\Omega_{0}}{\omega}\left(\frac{2 \omega \tilde{I}_{x}}{m_{e} c^{2}}-2 \gamma_{\text {init }}\right)=\varpi \varepsilon_{0},
$$

where $\varepsilon_{0}$ is a constant. Equations (4.2), (4.3) allow us to simplify equations (2.24), (2.27), (2.28) as:

$$
\left.\begin{array}{c}
\gamma=\left|\varpi-\frac{N_{\|}}{\sqrt{N_{\|}^{2}-1}} \sqrt{1+\varepsilon_{0} \varpi+\varpi^{2}}\right| \\
T=\frac{\gamma}{\sqrt{\gamma^{2}-1}} \oint\left(1-\frac{\varepsilon_{0}+2 \gamma}{\gamma^{2}-1} \varpi(s)\right)^{-1 / 2} \frac{\mathrm{d} s}{c} \\
\bar{r}=\frac{1}{2} \frac{N_{\|}}{N_{\|}^{2}-1}\left(\frac{\partial \ln \Omega_{0}}{\partial s / R}\right)\left(1-\gamma^{2}+\frac{\gamma^{2}}{N_{\|}^{2}}+\frac{\varpi}{N_{\|}^{2}} \frac{(\gamma-\varpi)^{2}}{(1-\varpi)}-\frac{\varpi^{2}}{N_{\|}^{2}}\right) \\
\langle\Delta \gamma\rangle=\sqrt{\frac{a \bar{r}}{\eta\left(N_{\|}^{2}-1\right)}} f_{S}(a), \quad a=\frac{2 e A_{0} \eta}{m_{e} c^{2}} \frac{\sqrt{\left(\varepsilon_{0}+2 \gamma\right) \varpi}}{\bar{r}},
\end{array}\right\}
$$

where $\eta=\omega R / c$.

The system (4.1), (4.4) is characterized by the following parameters: scale $R$, plasma density (defining the plasma frequency $\Omega_{\mathrm{pe}}$ ), dimensionless wave amplitude $2 e A_{e} / m_{e} c^{2}$, wave frequency $\omega$ and $\varepsilon_{0}$. For all following calculations, we use the Earth's dipole model with $R=R_{E} L\left(R_{E} \approx 6380 \mathrm{~km}\right.$ is the Earth's radius), a plasma density model (Sheeley et al. 2001) valid outside the plasmasphere in the inner magnetosphere providing $\Omega_{\mathrm{pe}}(L)$, and consider $L=4.5$ (the typical region of intense whistler mode chorus wave observations, see, e.g. Li et al. 2011a; Agapitov et al. 2013), $\omega=0.35 \Omega_{0}(0)$, for the chosen parameters $\eta \approx 2000,2 e A_{e} / m_{e} c^{2}=2 a_{0}(\lambda) / \eta$ (the corresponding wave magnetic field amplitude $\sim 500 \mathrm{pT}$ is typical for intense whistler mode chorus waves, e.g. Santolík et al. 2014) and function $a_{0}=\exp \left(-\left(\lambda-\lambda_{0}\right)^{2} / \delta \lambda^{2}\right)$ with $\lambda_{0}=25^{\circ}, \delta \lambda_{0}=12.5^{\circ}$ imitates a distribution of the wave intensity along magnetic field lines (e.g. Agapitov et al. 2013). For the above parameters, the main functions $N_{\|}, \partial \ln \Omega_{0} / \partial s / R$ and $s / R$ are displayed in figure $4(a)$ as a function of magnetic latitude $\lambda$.

The parameter $\varepsilon_{0}$ defines the range of energies and $\tilde{I}_{x}$ values considered for a particular solution. It is convenient to introduce the particle pitch angle $\alpha_{\text {eq }}$ (defined at $s=0$ ) and to rewrite $\varepsilon_{0}$ through initial energy and pitch angle. Using the definition of $I_{x}$, we can write $2 \tilde{I}_{x}=m_{e} c^{2}\left(\gamma_{\text {init }}^{2}-1\right) \sin ^{2} \alpha_{\text {eq }}$, and thus $\varepsilon_{0}=$ $\left(\gamma_{\text {init }}^{2}-1\right) \sin ^{2} \alpha_{\text {eq }} / \varpi(0)-2 \gamma_{\text {init }}$. The function $\varepsilon_{0}\left(\gamma_{\text {init }}, \alpha_{\text {eq }}\right)=$ const. corresponds to a certain relation between resonant particle energy and pitch angle, and this relation remains unchanged during the resonant interaction, i.e. $\varepsilon_{0}$ can be considered as an integral of particle motion. This integral represents a combination of the particle energy in the wave reference frame and the resonant condition (see analogies of this integral in, e.g. Shklyar 1981; Summers et al. 1998). Figure 4(b) shows $\varepsilon_{0}\left(\gamma_{\mathrm{init}}, \alpha_{\mathrm{eq}}\right)$. Choosing a particular $\varepsilon_{0}$, we can describe the evolution of a particle ensemble with initial energies and pitch angles defined by $\varepsilon_{0}\left(\gamma_{\text {init }}, \alpha_{\text {eq }}\right)$. The definition of the period $T$ (see (4.4)) determines the range of energies that must be considered for a given 

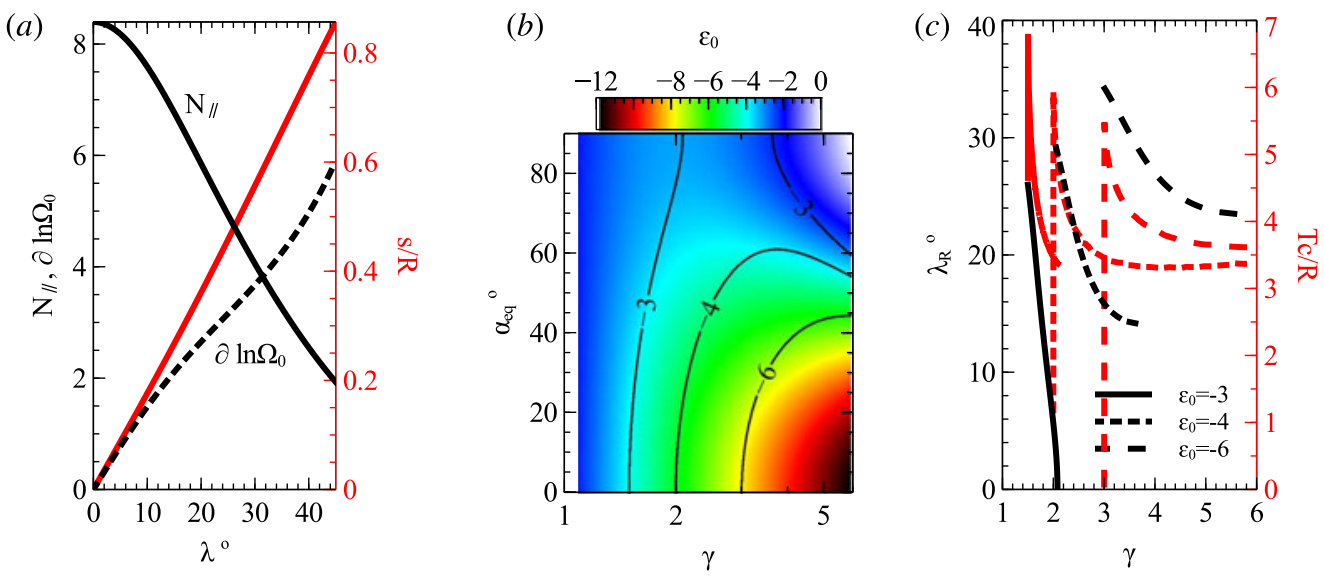

FIgURE 4. (a) Refractive index $N_{\|}$, derivative $\partial \ln \Omega_{0} / \partial s / R$ and field-aligned coordinate $s$ are shown as functions of $\lambda$. (b) System parameter $\varepsilon_{0}$ as a function of initial energy and pitch angle. $(c)$ Periods $T(\gamma)$ and resonant latitudes are shown for three values of $\varepsilon_{0}$ in the case of parallel whistler mode waves.
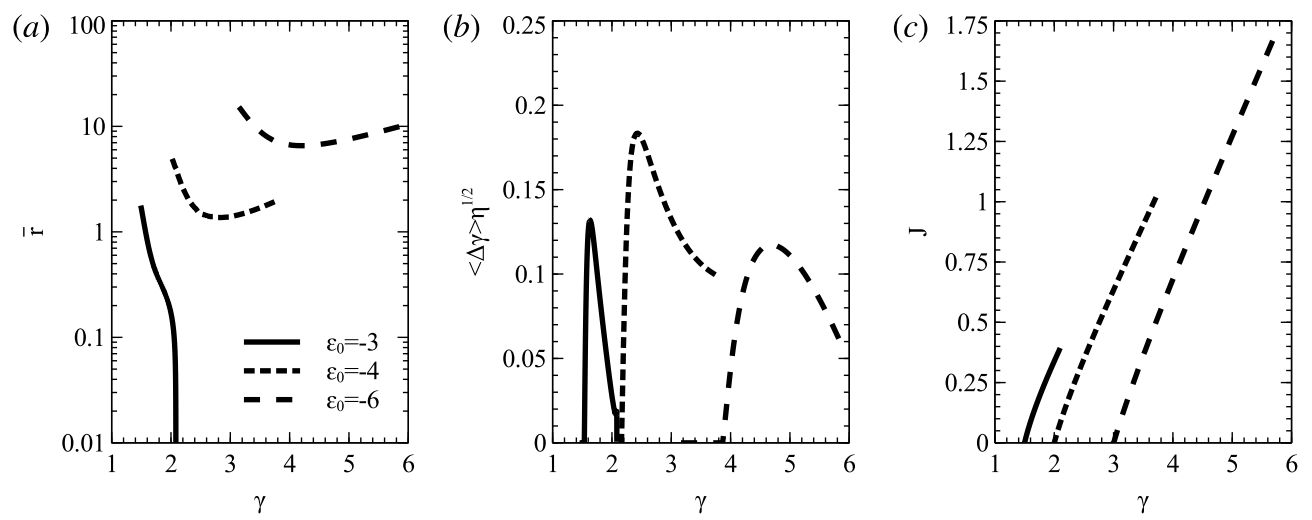

FIGURE 5. Functions $\bar{r}(\gamma)(a),\langle\Delta \gamma\rangle(\gamma)(b), J(\gamma)(c)$ are shown for three values of $\varepsilon_{0}$ in the case of parallel whistler mode waves.

$\varepsilon_{0}:\left(2 \varepsilon_{0}+2 \gamma\right) \varpi(0)<\gamma^{2}-1$. Figure $4(c)$ shows particle periods $T(\gamma)$ and resonant latitudes $\lambda_{R}$ (defined from the first equation of system (4.4)) for three different $\varepsilon_{0}$ values.

To construct (3.4) for the system (4.1), (4.4), we need first to define $\langle\Delta \gamma\rangle$, $J(\gamma)=\int T(\gamma) \mathrm{d} \gamma / 2 \pi$ (we use the renormalized $J c / R$ ) and $J=Y_{J}\left(J^{*}\right)$ projection. Equation (4.4) shows that $\langle\Delta \gamma\rangle$ depends on $\bar{r}(\gamma)$ presented in figure 5(a). Using this dependence, we calculate $\langle\Delta \gamma\rangle$ functions (see figure $5 b$ ) and define $V_{\gamma}=\langle\Delta \gamma\rangle / T(\gamma)$ and $V_{J}=V_{\gamma} T / 2 \pi$. The non-local (trapping term) of (3.4) is determined by the function $\gamma=Y\left(\gamma^{*}\right)$ shown in figure 6. A quite limited range of initial $\gamma^{*}$ is mapped to a wide range of final $\gamma$ as a result of the nonlinear interaction. The function $J(\gamma)$ shown in figure $5(c)$ allows us to transform the $\gamma$-dependence into a $J$-dependence and to solve (3.4).

Combining functions $J(\gamma),\langle\Delta \gamma\rangle, \gamma=Y\left(\gamma^{*}\right)$ and calculating the diffusion coefficient $D_{\gamma \gamma}=\left\langle(\Delta \gamma)^{2}\right\rangle / 2 T(\gamma)$ (see. e.g. Artemyev et al. 2016; Artemyev et al. 2017b), we 

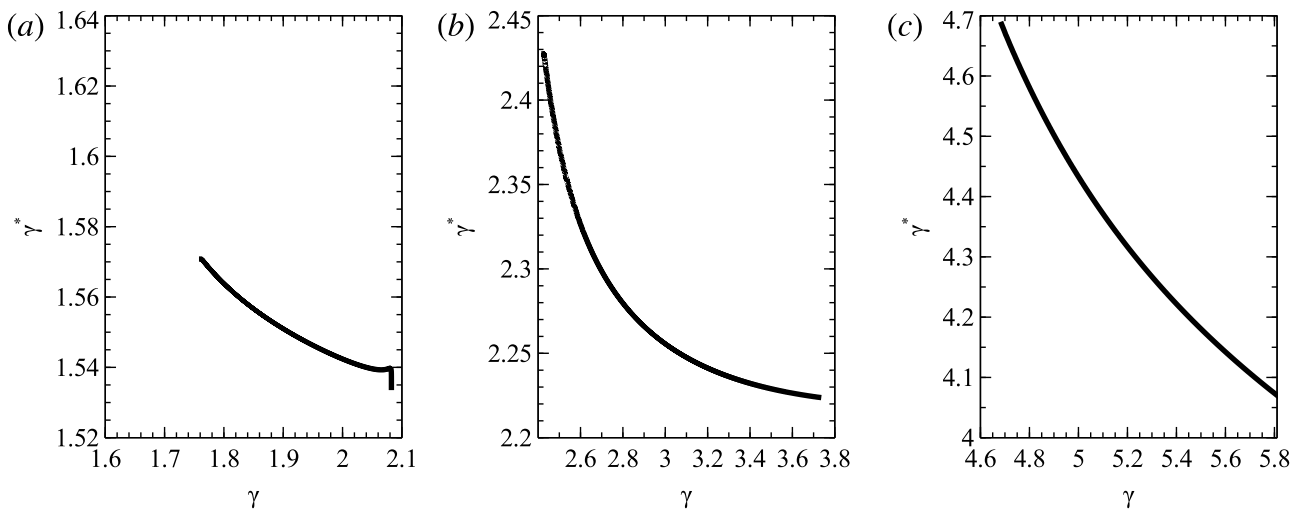

FIGURE 6. The case of parallel whistler mode waves. Dependencies $\gamma=Y\left(\gamma^{*}\right)$ for three $\varepsilon_{0}$ values: $\varepsilon_{0}=-3(a), \varepsilon_{0}=-4(b), \varepsilon_{0}=-6(c)$.

solve the kinetic equation for the $\Psi_{J}(J)$ distribution. Figure $7(b, d, f)$ shows the $\Psi(\gamma)=\Psi_{J}(J) T / 2 \pi$ distributions obtained for three different $\varepsilon_{0}$ values (the initial distribution $\Psi(\gamma) \sim \gamma^{-5}$ is shown by grey colour). Each $\varepsilon_{0}$ corresponds to some range of $\gamma, \alpha_{\text {eq }}$ (see figure $4 b$ ), and for different $\varepsilon_{0}$ the evolution of $\Psi(\gamma)$ reflects the dominance of particle trapping ( $\gamma$ increase) or drift $(\gamma$ decrease). We use boundary conditions $\partial \Psi / \partial J=0$ at the right-hand side (large $J$; large $\gamma$ ) and left-hand side (small $J$; small $\gamma$ ) boundaries. This condition allows a variation of boundary $\Psi$ values, but guarantees the absence of particle flux outside the $J$ range of the simulation. For all $\varepsilon_{0}$, the nonlinear wave-particle interaction results in the generation of a high-energy population due to trapping, which transports particles to higher $\gamma$. This population gradually drifts toward smaller energies, but this drift is weaker than the effect of trapping, i.e. the phase space density of high-energy particles grows. In all three cases, the distribution evolves toward a more uniform profile $\Psi(J)$ and we expect that after a sufficiently long time interval the final distribution will be $\Psi(J) \approx$ const., i.e. $\Psi$ will evolve toward the stationary solution of (3.4). The evolution of the entire particle distribution consists in $\Psi(\gamma)$ evolutions for a wide range of $\varepsilon_{0}$ values and, as a result, particle acceleration can be compensated by the deceleration of other particles, in such a way that the total energy variation of the entire particle ensemble can remain small (Shklyar 2011, 2017).

\section{Strongly oblique waves: Landau resonance}

In this section, we consider electrons in Landau resonance $n=0$ with strongly oblique whistler mode waves propagating with $\theta$ within one degree from the resonant cone angle $\theta_{r}=\arccos \left(\omega / \Omega_{0}\right)$ (Stix 1962). Therefore, the Hamiltonian $H_{1}$ from (2.13) takes the form

$$
\left.\begin{array}{c}
H_{1}=-e A_{0} h^{(0)} \sin \phi_{\|} \\
h^{(0)}=-\frac{\rho \Omega_{0}}{c \gamma} C J_{1}\left(k_{x} \rho\right)-\left(\frac{p_{z}}{\gamma m_{e} c}+C_{3}\right) \sin \theta J_{0}\left(k_{x} \rho\right),
\end{array}\right\}
$$

where $p_{z}=k_{z} I_{R}+p_{R}=\gamma / N_{\|}$. As in the previous section, we consider a magnetic field model with $\Omega=\Omega(0) \sqrt{1+3 \sin ^{2} \lambda} / \cos ^{6} \lambda$ and $\mathrm{d} s / \mathrm{d} \lambda=R \sqrt{1+3 \sin ^{2} \lambda} \cos \lambda$, where 

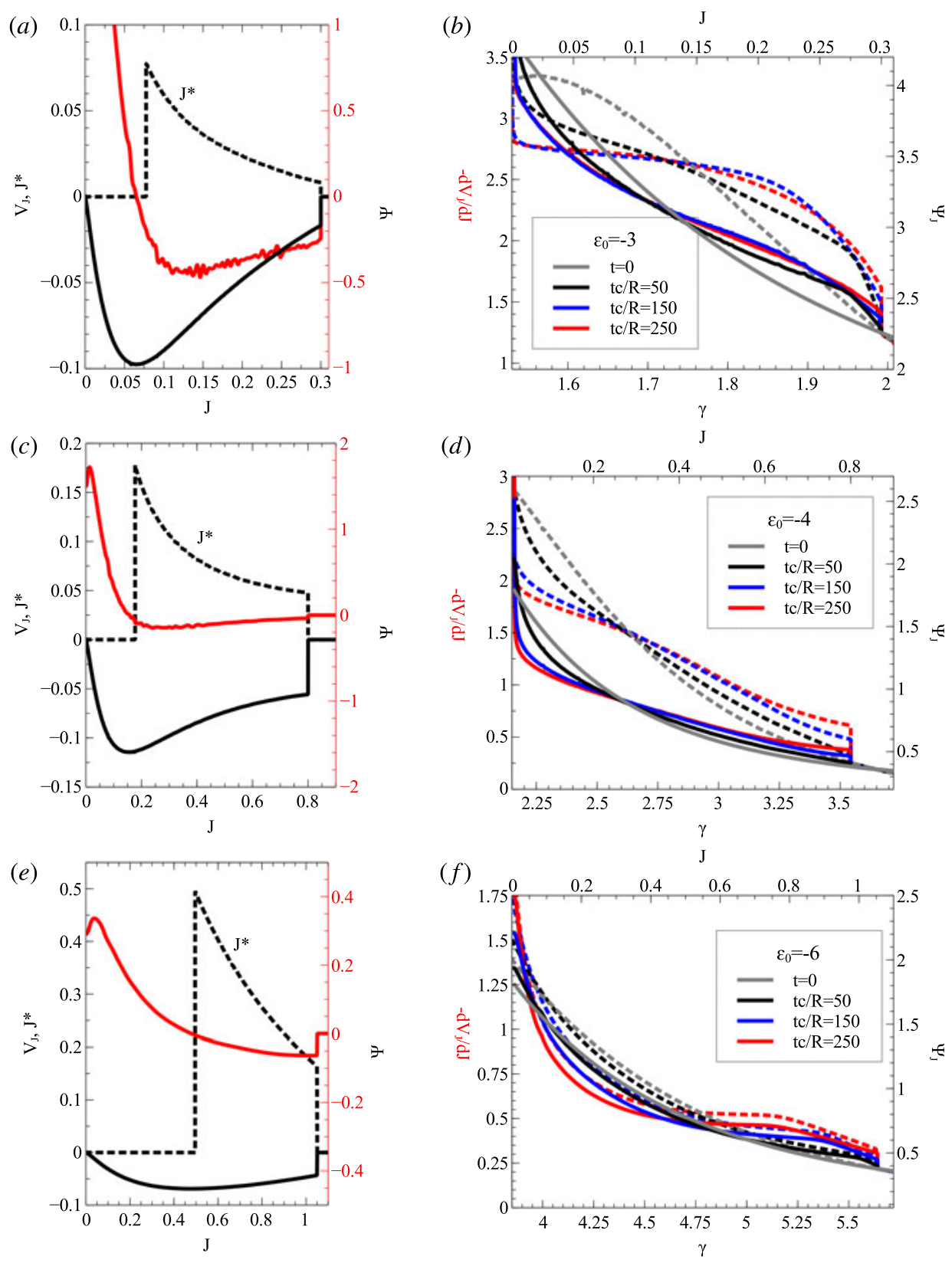

FIgURE 7. The case of parallel whistler mode waves. $(a, c, e)$ Show the distributions of $\eta^{-1 / 2} V_{J}(J), \quad-\eta^{-1 / 2} \mathrm{~d} V_{J} / \mathrm{d} J$, and $J^{*}(J)$ (inverse $Y_{J}\left(J^{*}\right)$ function). (b,d,f) Show the distributions $\Psi_{J}(J)$ (dashed) and $\Psi(\gamma)$ (solid) at three different times $t c / R$. The data are plotted for three different $\varepsilon_{0}$ values. 
$R$ is the spatial scale of magnetic field inhomogeneity. Using $\varpi=\Omega_{0}(s) / \omega$ and a simplified dispersion relation valid for obliquely propagating waves (Stix 1962), we write:

$$
N_{\|}=\frac{\Omega_{\mathrm{pe}} \cos \theta}{\omega \sqrt{\varpi \cos \theta-1}}, \quad \frac{\partial \ln N_{\|}}{\partial \ln \Omega_{0}}=-\frac{1}{2} \frac{\varpi \cos \theta}{\varpi \cos \theta-1},
$$

where $\Omega_{\mathrm{pe}}=$ const. is the plasma frequency. Using $\omega_{n}=0$, we rewrite $\varepsilon=\beta_{\perp}^{2}-2 \gamma_{\text {init }} \omega_{n}$ as:

$$
\varepsilon=\beta_{\perp}^{2}=\frac{\Omega_{0}}{\omega} \frac{2 \omega \tilde{I}_{x}}{m_{e} c^{2}}=\varpi \varepsilon_{0},
$$

where $\varepsilon_{0}$ is a constant. Equations (5.2), (5.3) allow us to simplify (2.24), (2.25), (2.28):

$$
\left.\begin{array}{c}
\gamma=\frac{N_{\|}}{\sqrt{N_{\|}^{2}-1}} \sqrt{1+\varepsilon_{0} \varpi}, \quad T=\frac{\gamma}{\sqrt{\gamma^{2}-1}} \oint\left(1-\frac{\varepsilon_{0} \varpi(s)}{\gamma^{2}-1}\right)^{-1 / 2} \frac{\mathrm{d} s}{c} \\
\bar{r}=\frac{1}{2} \frac{N_{\|}}{N_{\|}^{2}-1}\left(\frac{\partial \ln \Omega_{0}}{\partial s / R}\right)\left(1-\gamma^{2}-\frac{\gamma^{2}}{N_{\|}^{2}} \frac{1}{\varpi \cos \theta-1}\right) \\
\langle\Delta \gamma\rangle=\sqrt{\frac{a \bar{r}}{\eta\left(N_{\|}^{2}-1\right)} f_{S}(a), \quad a=\frac{2 e A_{0} \eta}{m_{e} c^{2}} \frac{\sqrt{\varepsilon_{0} \varpi} h^{(0)}}{\bar{r}}, \quad k_{x} \rho=\sqrt{\varepsilon_{0} \varpi} \frac{N_{\|}}{\varpi} \frac{\sin \theta}{\cos \theta}} \\
h^{(0)}=-\frac{\sqrt{\varepsilon_{0} \varpi}}{\gamma} C J_{1}\left(k_{x} \rho\right)-\left(N_{\|}+C_{3}\right) \sin \theta J_{0}\left(k_{x} \rho\right),
\end{array}\right\}
$$

where $\eta=\omega R / c$. Similarly to (4.1), (4.4), equations (5.4) include several parameters: we use the same scale $R, \omega$ and plasma density $\Omega_{\mathrm{pe}}$ as for parallel propagating wave (and, thus $\eta \approx 2000$ ), whereas the dimensionless wave amplitude is taken as $2 e A_{e} / m_{e} c^{2}=1.5 a_{0}(\lambda) / \eta$ (the corresponding wave magnetic field amplitude $\sim 150 \mathrm{pT}$ is typical of intense highly oblique whistler mode chorus waves, e.g. Cully et al. 2008; Wilson et al. 2011; Agapitov et al. 2014). Figure 8(a) shows the parallel refractive index $N_{\|}$and $\cos \theta$ along the magnetic latitude $\lambda$. As latitude increases, $\theta$ increases too, whereas $\cos \theta$ decreases to zero.

Equation (5.3) shows that the parameter $\varepsilon_{0}=\left(\gamma_{\text {init }}^{2}-1\right) \sin ^{2} \alpha_{\text {eq }} / \varpi(0)$ is positive and equal to the initial $I_{x} / \varpi(0)$ value (i.e. for Landau resonance the $\varepsilon_{0}$ conservation means the conservation of the magnetic moment $I_{x}$ ). For Landau resonance $n=0$, the wave phase does not depend on the phase conjugated to $I_{x}$, and thus $I_{x}$ is conserved for all particles (see Hamiltonian (2.14)). It is convenient to introduce the particle pitch angle $\alpha_{\text {eq }}$ (defined at $s=0$ ) and to rewrite $\varepsilon_{0}$ as a function of initial energy and pitch angle. Figure $8(b)$ shows $\varepsilon_{0}\left(\gamma_{\text {init }}, \alpha_{\text {eq }}\right)$. For calculations within this section, we use three $\varepsilon$ values: $0.5,1$ and 2 . Using these $\varepsilon_{0}$, we plot in figure $4(c)$ the particle periods $T(\gamma)$ and resonant latitudes $\lambda_{R}$ (defined from the first equation of system (5.4)).

Using (5.4), we define the functions $\bar{r}(\gamma)$ and $J(\gamma)=\int T \mathrm{~d} \gamma / 2 \pi$ (we use the renormalized $J=J c / R$ ) and plot them in figure 9. These functions have similar profiles as in the case of cyclotron resonance with parallel propagating waves (see figure 5). Unlike $\bar{r}(\gamma), J(\gamma)$, however, the function $\langle\Delta \gamma\rangle$ has a much more complicated profile for Landau resonance (compare the central panels of figures 5 and 9). The effective wave amplitude given by (5.4) depends on a combination of Bessel functions $J_{0}$, $J_{1}$, and thus this amplitude oscillates along the resonant particle trajectory (with $\gamma$ ). Such oscillations result in several maxima/minima of $\langle\Delta \gamma\rangle$, i.e. the particles can 

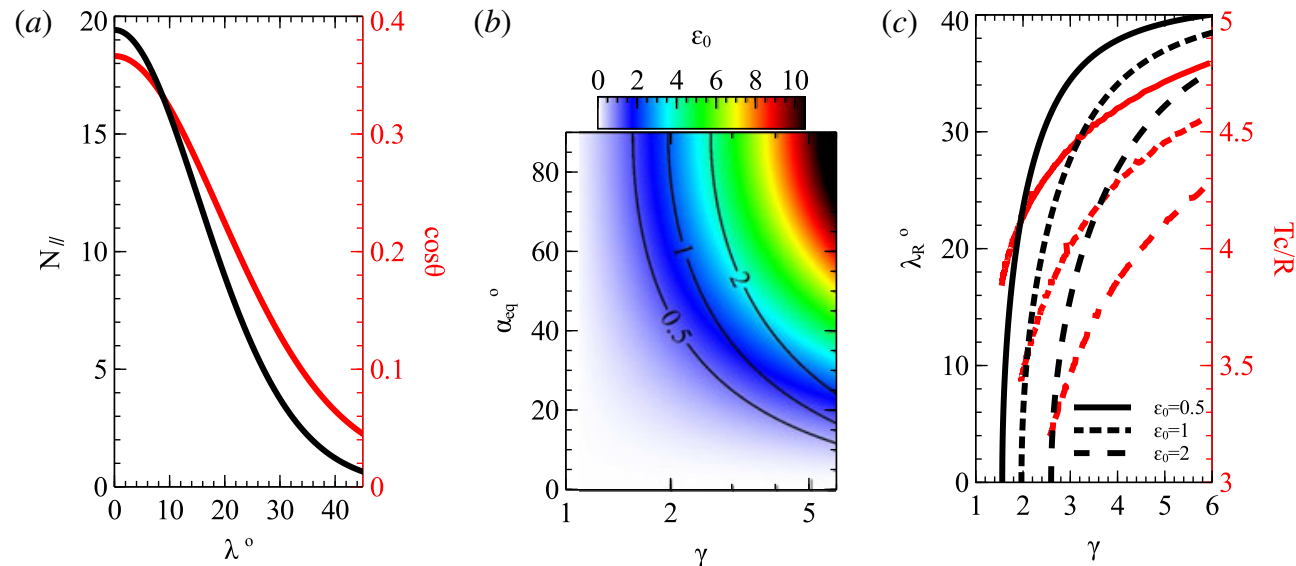

FIgURE 8. The case of very oblique whistler mode waves. (a) Refractive index $N_{\|}$and $\cos \theta$ are shown as functions of $\lambda$. (b) System parameter $\varepsilon_{0}$ as a function of initial energy and pitch angle. (c) Periods $T(\gamma)$ and resonant latitudes for three values of $\varepsilon_{0}$.
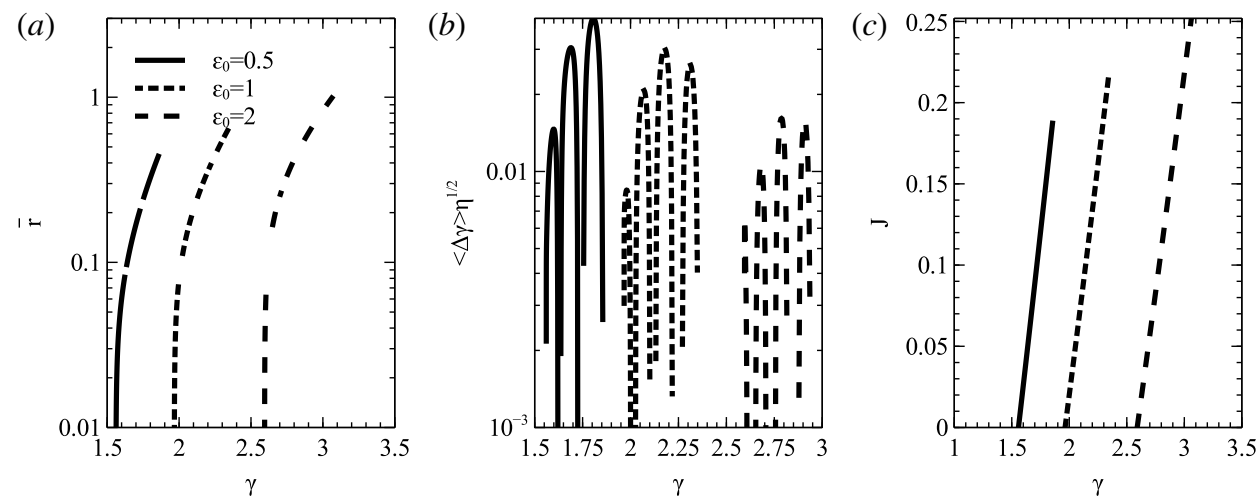

FIGURE 9. Functions $\bar{r}(\gamma)(a),\langle\Delta \gamma\rangle(\gamma)(b), J(\gamma)(c)$ for three different values of $\varepsilon_{0}$ in the case of very oblique whistler mode waves.

get trapped in many regions (with trapping condition $\left.\mathrm{d} S_{\text {sep }} / \mathrm{d} \gamma=\mathrm{d}\langle\Delta \gamma\rangle / \mathrm{d} \gamma\right\rangle 0$, see $(2.29 a, b))$ separated in $\gamma$ space by regions where particle escape from trapping (where $\left.\mathrm{d} S_{\text {sep }} / \mathrm{d} \gamma=\mathrm{d}\langle\Delta \gamma\rangle / \mathrm{d} \gamma<0\right)$.

Figure 10 demonstrates that the $Y\left(\gamma^{*}\right)$ function contains several regions with very sharp gradients (several almost vertical lines). Thus, all particles trapped within a wide energy $\gamma^{*}$ range (within a region with $\mathrm{d}\langle\Delta \gamma\rangle / \mathrm{d} \gamma>0$ ) will escape at the next (after the trapping region) decrease of $\langle\Delta \gamma\rangle$ with almost the same energy $\gamma=Y\left(\gamma^{*}\right)$. Using the functions shown in figures 9 and 10, we can calculate all coefficients of (3.4).

Next, using functions from figures 9 and 10 we solve the kinetic equation for the $\Psi_{J}(J)$ distribution and then recalculate from this distribution the $\Psi(\gamma)=\Psi_{J}(J) T / 2 \pi$ distribution. Figure $11(b, d, f)$ shows the $\Psi(\gamma), \Psi_{J}(J)$ distributions obtained for three $\varepsilon_{0}$ values (the initial distribution $\Psi(\gamma) \sim \gamma^{-5}$ is shown by grey colour). For all $\varepsilon_{0}$ values, the evolution of $\Psi(\gamma)$ has rather similar characteristics. There are several regions where particles trapped with a smaller energy are released from the resonance with the energy increase. This transport results in the formation of local enchantments 
(a)

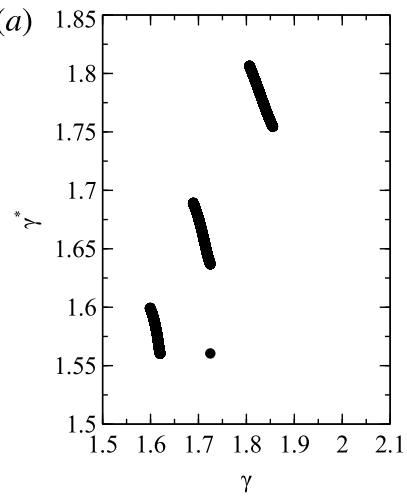

(b)

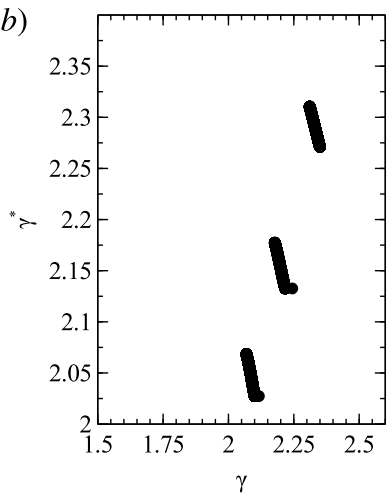

(c)

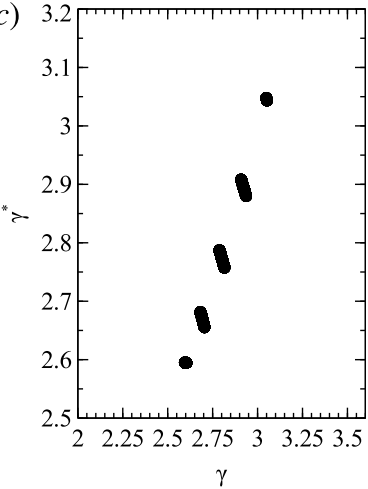

FIGURE 10. Dependencies $\gamma=Y\left(\gamma^{*}\right)$ for three $\varepsilon_{0}$ values in the case of very oblique whistler mode waves: $\varepsilon_{0}=0.5(a), \varepsilon_{0}=1(b), \varepsilon_{0}=2(c)$.

of $\Psi(\gamma)$ (the location of these enchantments is different for different $\varepsilon_{0}$ ). After their formation, these local enchantments of $\Psi(\gamma)$ evolve in time: the drift toward smaller energy evacuates particles and forms a shoulder on the left side of each initial distribution enchantment. Such a competition between particle evacuation due to trapping and particle drift to smaller energies due to scattering should finally results in the formation of uniform distributions within energy ranges bounded by values corresponding to $\mathrm{d} V / \mathrm{d} J$ peaks. (Note that singular-like peaks at the boundaries of each evolution region are due to the absence of diffusion in these locations $\left(\partial D_{J J} / \partial J\right.$ is much weaker than $V_{J}$ ); these peaks would quickly get smoothed out in a more realistic system with non-monochromatic waves.) Moreover, when considering the global particle distribution, consisting in $\Psi(\gamma)$ with a large range of $\varepsilon_{0}$ values, the different effects should mingle and these individual peaks should get smoothed out and simply form a high-energy tail to the particle population. At the right-hand side of each peak in $\Psi(\gamma)$, a local minimum is formed. This minimum is due to trapped particle transport to higher energy: the efficiency of trapping $\sim \mathrm{d} V_{J} / \mathrm{d} J \sim \mathrm{d} V / \mathrm{d} \gamma$ has a large maximum around these minima due to the rapid growth of $\langle\Delta \gamma\rangle$ there (see figure $10 b$ ).

\section{Discussion and conclusion}

Our description of nonlinear resonant interactions of electrons with whistler mode waves relies on the full kinetic equation (3.4) derived for coherent whistler waves (such as observed in the radiation belts, see, e.g. Li et al. 2011b; Agapitov et al. 2015). The applicability of the quasi-linear theory in the case of coherent narrow band waves with isolated resonances can be justified by the background magnetic field inhomogeneity (in the absence of a broad wave spectrum the resonance width is defined by the wave spatial dispersion, see, e.g. Karpman 1974; Le Queau \& Roux 1987; Demekhov et al. 2000). The applicability of either the quasi-linear theory or nonlinear wave-particle interaction models is determined by the wave amplitude level (see quasi-linear diffusion coefficient calculations for coherent narrow band waves in an inhomogeneous plasma in, e.g. Albert 2010). Thus, we can compare the respective time scales of evolution of the electron distribution due to quasi-linear scattering versus that due to the nonlinear interaction described by equation (3.4). In addition to the diffusion term $\partial\left(D_{J J} \partial \Psi / \partial J\right) / \partial J$, this kinetic equation contains a 

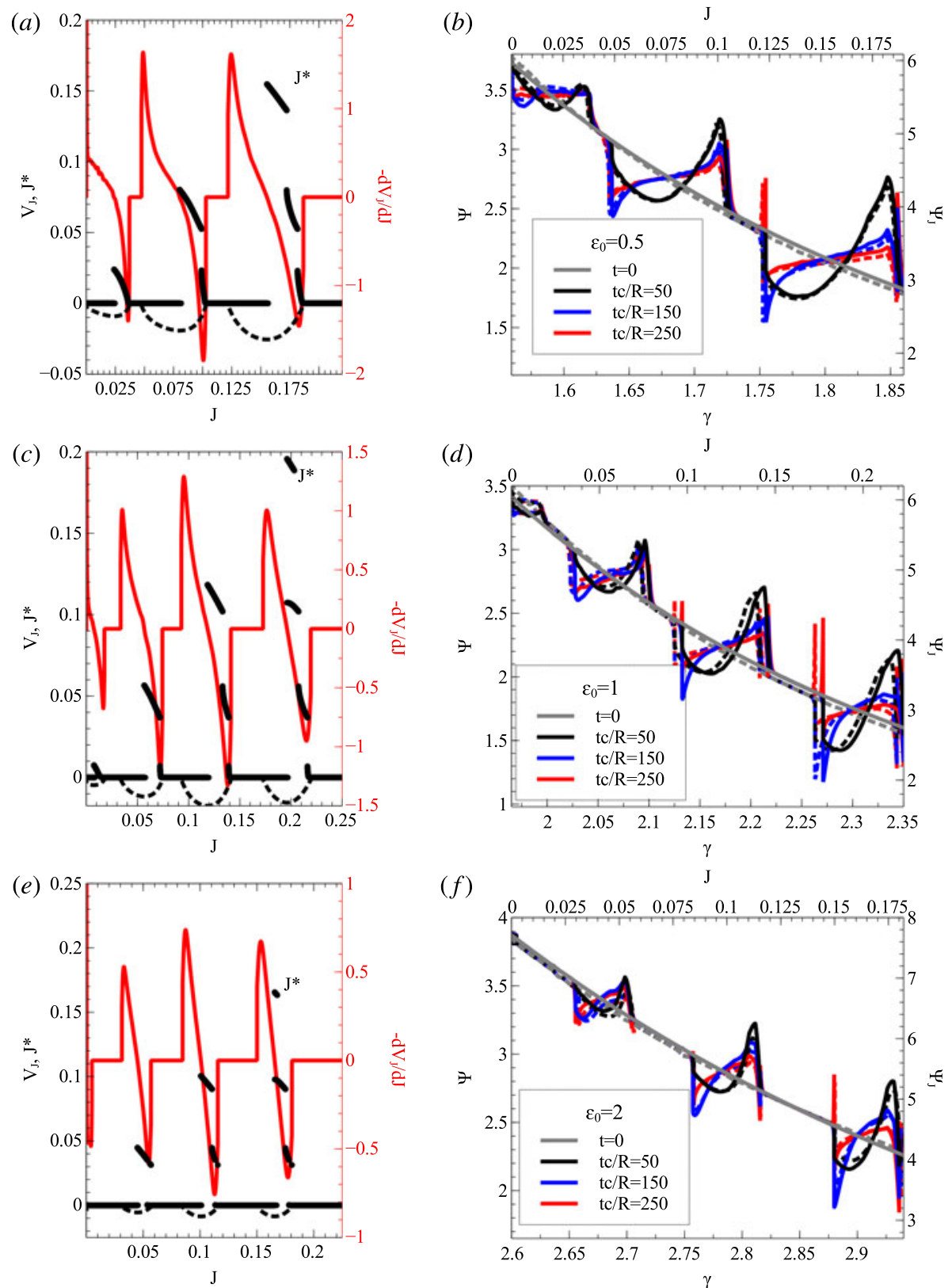

FIgURE 11. (a,c,e) Show distributions of $\eta^{-1 / 2} V_{J}(J),-\eta^{-1 / 2} \mathrm{~d} V_{J} / \mathrm{d} J$ and $J^{*}(J)$ (inverse $Y_{J}\left(J^{*}\right)$ function). $(b, d, f)$ Show the distributions $\Psi_{J}(J)$ (dashed) and $\Psi(\gamma)$ (solid) at three different times $t c / R$. Data are plotted for three $\varepsilon_{0}$ values in the case of highly oblique whistler mode waves.

drift term $\sim V_{J} \partial \Psi / \partial J$ and a non-local transport term $\sim\left(\partial V_{J} / \partial J\right)\left(\Psi-\Psi^{*}\right)$. These two terms are proportional to $V_{J} \sim\langle\Delta \gamma\rangle \sim \sqrt{e A_{0} / m_{e} c^{2}}$ (see (2.28)). The diffusion term $D_{J J}$ is of the order of $e A_{0} / m_{e} c^{2}$ in the nonlinear regime $(\langle\Delta \gamma\rangle \neq 0)$ and it is approximately $\left(e A_{0} / m_{e} c^{2}\right)^{2}$ in the quasi-linear regime (Kennel \& Engelmann 1966; 
Karpman 1974; Albert 2010). The ratio $e A_{0} / m_{e} c^{2} \ll 1$ is a small parameter in this system, and nonlinear terms $\sqrt{e A_{0} / m_{e} c^{2}}$ are much larger than the diffusion term in the kinetic equation (3.4). Thus, in the nonlinear regime (when $\langle\Delta \gamma\rangle \neq 0$ or $a>1$ in (2.28)), the particle dynamics is mostly defined by the competition between trapping transport (energy increase) and drift (energy decrease). Both processes are essentially non-diffusive, and the $\Psi$ evolution can consist of the formation of localized (in energy space) structures and their following drifts (see examples in figures 7 and 11 and (Leoncini, Vasiliev \& Artemyev 2018)). This allows us to apply (3.4) for the description of many coherent phenomena in the Earth's radiation belts as well as in other space plasma systems where the wave amplitude is sufficiently high: the formation of intense electron precipitation bursts into the atmosphere (e.g. Albert \& Bortnik 2009; Omura \& Zhao 2012, 2013), rapid electron acceleration (Agapitov et al. 2015; Mozer et al. 2016; Foster et al. 2017), rapid isotropization of electron distributions (Agapitov et al. 2016; Mourenas et al. 2016) and other applications (see discussion in Artemyev et al. 2017b). At the present time, the effects related to nonlinear wave-particle interactions are basically described using a test-particle approach (Bortnik et al. 2008; Yoon et al. 2013; Nunn \& Omura 2015) or an analogue to (3.4) with coefficients derived from test-particle simulations (Omura et al. 2015; Hsieh \& Omura 2017b). Therefore, the approach proposed here has a very wide range of potential applications, although great care should be exerted when attempting to generalize or modify (3.4) for particular applications to real plasma systems.

In contrast to the quasi-linear theory, which provides a diffusion equation with diffusion rates depending only on the wave average intensity (Drummond \& Pines 1962; Vedenov, Velikhov \& Sagdeev 1962), the nonlinear kinetic equation (3.4) requires detailed information about different characteristics of intense waves: the distribution of wave amplitudes instead of the average intensity, their spectrum properties (e.g. the wave sweep rate $\partial \omega / \partial t$, see Demekhov et al. 2009; Hsieh \& Omura 2017a), the wave coherency (see Artemyev et al. 2012; Tao et al. $2012 b$ ) as well as information on the presence or not of resonant and non-resonant electromagnetic noise and satellite waves destroying resonant interaction (Brinca 1980; Nunn 1986; Artemyev et al. 2015). All these requirements significantly complicate the practical use of (3.4) for massive long-term calculations of the electron distribution, which rely today only on quasi-linear diffusion models (e.g. see Glauert et al. 2014; Drozdov et al. 2015; Ma et al. 2016). Therefore, we suggest that (3.4) can be useful for the determination of the role played by nonlinear wave-particle interaction in short-term events (but including many resonant interactions), but that this equation should be further modified (simplified) to be included into global codes. Let us discuss such possible approaches.

Firstly, any modulation of the wave (e.g. loss of wave coherency, see Tsurutani et al. 2011; Santolík et al. 2014; Agapitov et al. 2017; Turner et al. 2017) significantly reduces the efficiency of trapped particle acceleration (see Artemyev et al. 2012; Tao et al. 2012b, 2013). This can result in smaller individual acceleration jumps, potentially allowing us to simplify considerably the non-local transport term. The corresponding simplified kinetic equation might be more easily evaluated for a broad range of wave characteristics (e.g. Albert 2002; Shklyar \& Matsumoto 2009; Artemyev et al. 2013). The effect of wave modulation (presence of short wave packets instead of infinite plane waves) should significantly constrain the applicability of nonlinear wave-particle interaction models. Waves with highly modulated amplitudes should resonate with particles in a regime closer to quasi-linear scattering. Thus, there is a 
transition between nonlinear interaction with coherent waves and the almost stochastic diffusion supported by non-coherent (deeply modulated) wave packets. Although both limiting regimes of nonlinear interaction and quasi-linear diffusion have been well investigated, the transient regime where particles interact with intense short wave packets requires further consideration.

Secondly, equation (3.4) describes the $\Psi$ evolution for a particular $\varepsilon_{0}$ which depends on the wave frequency, i.e. this equation is written for only one wave. Dealing with several intense waves (but still without resonance overlapping, see Shapiro \& Sagdeev 1997; Shklyar \& Matsumoto 2009), we need to use several copies of (3.4) with different $\varepsilon_{0}$, but the same distribution $\Psi$. Such a modification significantly complicates the evaluation of non-local terms. A possible solution of this problem is to rewrite $\Psi-\Psi^{*}$ in an integral form where wave characteristics are included in an integral operator (see, e.g. Artemyev et al. 2016). Then, the averaging over the wave parameter (e.g. frequency) distribution function can be performed for this integral operator which does not depend on $\Psi$. Thus, instead of $\Psi^{*}$, equation (3.4) will contain a $\int K\left(J, J^{*}\right) \Psi(J) \mathrm{d} J$ term where the integral kernel $K$ equals the Dirac delta function $\delta\left(J-J^{*}\right)$ for a single wave, but is more complicated for a wave ensemble.

Both discussed modifications (expansion of non-local term in (3.4) or rewriting of this term in an integral form) can potentially help to generalize the non-local kinetic equation (3.4) to meet requirements for practical modelling of realistic plasma systems.

Nonlinear wave-particle interactions in the presence of strong wave amplitude modulation and resonance destruction, can still lead to an evolution of the particle velocity distribution similar as in the quasi-linear diffusive regime. Spatial variations of the wave intensity (partially due to the multiplication factor depending on Bessel functions for oblique waves, see (5.4)) results in a mixing of trapped and scattered particles during time interval of the order of one bounce period (one period of $\left(z, p_{z}\right)$ oscillations). The fine balance between energy changes of the scattered particles and the phase space filled by trapped particles (both these quantities are approximately $S_{\text {res }}$ ) guarantees that trapping and nonlinear scattering will eventually compensate each other over very long time intervals (e.g. Solovev \& Shklyar 1986). Then, the second-order effect of particle diffusion will eventually control the evolution of the particle distribution function. However, the time scale for the eventual establishment of such a quasi-diffusion regime is not so small, i.e. there is a quite prolonged time interval when different particle populations, separated in phase space, experience trapping and scattering. Thus, there is a time interval (containing many resonances) when trapping and nonlinear scattering effects are not totally in balance, allowing significant nonlinear modifications of the particle distribution. The equations derived in the present study describe the evolution of the particle distribution during this time interval. Moreover, in realistic situations where there is a particle sink in some region of phase space (the loss cone for radiation belt electrons) such that particles get very quickly lost from the system when they reach this region, or when the wave parameters (frequency, amplitude) vary sufficiently fast (over minutes, i.e. over $10^{2}-10^{3}$ bounce periods) in such a way that nonlinear interactions only exist over a relatively limited time period, then there can be a non-null net effect of nonlinear wave-particle interaction, very different from the effects of diffusion. The nonlinear transport of particles into a region of space phase where the probability of nonlinear interaction is very small (much smaller than before) can further help to get a net nonlinear effect.

In conclusion, we have derived the non-local kinetic equation describing nonlinear resonant interactions of charged particles with intense whistler mode waves propagating 
with an arbitrary wave normal angle. This equation generalizes a simpler form of non-local kinetic equation initially derived for electrostatic parallel propagating waves in Artemyev et al. (2017b). Using the derived equation, we describe the evolution of the electron distribution function $\Psi(\gamma)$ in a system with either an intense parallel propagating whistler model wave or a highly oblique whistler mode wave. This evolution consists in a competition between two nonlinear resonant processes: trapped particle transport and scattered particle drift in energy space. We have demonstrated how the wave properties influence the principal character of the nonlinear interaction, leading to either formation of high-energy electron populations or an effective deceleration of high-energy electrons. The proposed approach can be applied to describe a wide range of resonant interactions in space plasma systems.

\section{Acknowledgements}

Work of A.V.A., A.I.N., and A.A.V. was supported by Russian Scientific Fund (project no. 14-12-00824).

\section{REFERENCES}

Agapitov, O., Artemyev, A., Krasnoselskikh, V., Khotyaintsev, Y. V., Mourenas, D., Breuillard, H., Balikhin, M. \& Rolland, G. 2013 Statistics of whistler mode waves in the outer radiation belt: Cluster STAFF-SA measurements. J. Geophys. Res. 118, 3407-3420.

Agapitov, O., Artemyev, A., Mourenas, D., Krasnoselskikh, V., Bonnell, J., Le Contel, O., Cully, C. M. \& ANGelopoulos, V. 2014 The quasi-electrostatic mode of chorus waves and electron nonlinear acceleration. J. Geophys. Res. 119, 1606-1626.

Agapitov, O. V., Artemyev, A. V., Mourenas, D., Mozer, F. S. \& Krasnoselskikh, V. 2015 Nonlinear local parallel acceleration of electrons through Landau trapping by oblique whistler mode waves in the outer radiation belt. Geophys. Res. Lett. 42, 10.

Agapitov, O., Blum, L. W., Mozer, F. S., Bonnell, J. W. \& Wygant, J. 2017 Chorus whistler wave source scales as determined from multipoint Van Allen Probe measurements. Geophys. Res. Lett. 44, 2634-2642.

Agapitov, O., Krasnoselskikh, V., Zaliznyak, Y., Angelopoulos, V., Le Contel, O. \& RoLlAND, G. 2011 Observations and modeling of forward and reflected chorus waves captured by THEMIS. Ann. Geophys. 29, 541-550.

Agapitov, O. V., Mourenas, D., Artemyev, A. V.\& Mozer, F. S. 2016 Exclusion principle for very oblique and parallel lower band chorus waves. Geophys. Res. Lett. 43 (21), 11112-11120.

Albert, J. M. 2002 Nonlinear interaction of outer zone electrons with VLF waves. Geophys. Res. Lett. 29, 1275.

Albert, J. M. 2010 Diffusion by one wave and by many waves. J. Geophys. Res. 115, A14.

Albert, J. M. \& BORTNIK, J. 2009 Nonlinear interaction of radiation belt electrons with electromagnetic ion cyclotron waves. Geophys. Res. Lett. 36, 12110.

Albert, J. M., TAO, X. \& BortniK, J. 2013 Aspects of nonlinear wave-particle interactions. In Dynamics of the Earth's Radiation Belts and Inner Magnetosphere (ed. D. Summers, I. U. Mann, D. N. Baker \& M. Schulz), Geophysical Monograph Series. American Geophysical Union.

Albert, J. M. \& Young, S. L. 2005 Multidimensional quasi-linear diffusion of radiation belt electrons. Geophys. Res. Lett. 32, 14110.

Arnold, V. I., Kozlov, V. V. \& NeIshtadt, A. I. 2006 Mathematical Aspects of Classical and Celestial Mechanics, 3rd edn. Springer.

Artemyev, A., Krasnoselskikh, V., Agapitov, O., Mourenas, D. \& Rolland, G. 2012 Non-diffusive resonant acceleration of electrons in the radiation belts. Phys. Plasmas 19, 122901. 
Artemyev, A. V., Mourenas, D., Agapitov, O. V., Vainchtein, D. L., Mozer, F. S. \& KRASNOSELSKIKH, V. V. 2015 Stability of relativistic electron trapping by strong whistler or electromagnetic ion cyclotron waves. Phys. Plasmas 22, 082901.

Artemyev, A. V., Neishtadt, A. I., Vasiliev, A. A.\& Mourenas, D. 2016 Kinetic equation for nonlinear resonant wave-particle interaction. Phys. Plasmas 23 (9), 090701.

Artemyev, A. V., Neishtadt, A. I., Vasiliev, A. A. \& Mourenas, D. $2017 a$ Kinetic equation for systems with resonant captures and scatterings. Preprint, arXiv:1710.04489.

Artemyev, A. V., Neishtadt, A. I., Vasiliev, A. A. \& Mourenas, D. $2017 b$ Probabilistic approach to nonlinear wave-particle resonant interaction. Phys. Rev. E 95 (2), 023204.

Artemyev, A. V., Vasiliev, A. A., Mourenas, D., Agapitov, O. \& Krasnoselskikh, V. 2013 Nonlinear electron acceleration by oblique whistler waves: Landau resonance versus cyclotron resonance. Phys. Plasmas 20, 122901.

Artemyev, A. V., Vasiliev, A. A., Mourenas, D., Agapitov, O., Krasnoselskikh, V., Boscher, D. \& Rolland, G. 2014 Fast transport of resonant electrons in phase space due to nonlinear trapping by whistler waves. Geophys. Res. Lett. 41, 5727-5733.

BELL, T. F. 1984 The nonlinear gyroresonance interaction between energetic electrons and coherent VLF waves propagating at an arbitrary angle with respect to the earth's magnetic field. J. Geophys. Res. 89, 905-918.

BORTNIK, J., INAN, U. S. \& BELL, T. F. 2006 Landau damping and resultant unidirectional propagation of chorus waves. Geophys. Res. Lett. 33, L03102.

BORTNIK, J., ThORNe, R. M. \& INAN, U. S. 2008 Nonlinear interaction of energetic electrons with large amplitude chorus. Geophys. Res. Lett. 35, 21102.

Breuillard, H., Zaliznyak, Y., Agapitov, O., Artemyev, A., Krasnoselskikh, V. \& Rolland, G. 2013 Spatial spreading of magnetospherically reflected chorus elements in the inner magnetosphere. Ann. Geophys. 31, 1429-1435.

BRINCA, A. L. 1980 On the evolution of the geomagnetospheric coherent cyclotron resonance in the midst of noise. J. Geophys. Res. 85, 4711-4714.

Cattell, C., Wygant, J. R., Goetz, K., Kersten, K., Kellogg, P. J., von Rosenvinge, T., BAle, S. D., Roth, I., Temerin, M., Hudson, M. K. et al. 2008 Discovery of very large amplitude whistler-mode waves in Earth's radiation belts. Geophys. Res. Lett. 35, 1105.

Chen, L., Thorne, R. M., LI, W. \& Bortnik, J. 2013 Modeling the wave normal distribution of chorus waves. J. Geophys. Res. 118, 1074-1088.

Cully, C. M., Bonnell, J. W. \& Ergun, R. E. 2008 THEMIS observations of long-lived regions of large-amplitude whistler waves in the inner magnetosphere. Geophys. Res. Lett. 35, 17.

Demekhov, A. G., Trakhtengerts, V. Y., Hobara, Y. \& Hayakawa, M. 2000 Cyclotron amplification of whistler waves by nonstationary electron beams in an inhomogeneous magnetic field. Phys. Plasmas 7, 5153-5158.

Demekhov, A. G., Trakhtengerts, V. Y., Rycroft, M. \& Nunn, D. 2009 Efficiency of electron acceleration in the Earth's magnetosphere by whistler mode waves. Geomagn. Aeron. 49, 24-29.

Demekhov, A. G., Trakhtengerts, V. Y., Rycroft, M. J. \& Nunn, D. 2006 Electron acceleration in the magnetosphere by whistler-mode waves of varying frequency. Geomagn. Aeron. 46, 711-716.

Dolgopyat, D. 2012 Repulsion from resonances. In Memoires De La Societe Mathematique De France, vol. 128. Amer Mathematical Society.

Drozdov, A. Y., Shprits, Y. Y., Orlova, K. G., Kellerman, A. C., Subbotin, D. A., Baker, D. N., Spence, H. E. \& Reeves, G. D. 2015 Energetic, relativistic, and ultrarelativistic electrons: comparison of long-term VERB code simulations with Van Allen Probes measurements. J. Geophys. Res. 120, 3574-3587.

Drummond, W. E. \& Pines, D. 1962 Nonlinear stability of plasma oscillations. Nucl. Fusion Suppl. 3, 1049-1058.

Foster, J. C., Erickson, P. J., Omura, Y., Baker, D. N., Kletzing, C. A. \& Claudepierre, S. G. 2017 Van allen probes observations of prompt mev radiation belt electron acceleration in nonlinear interactions with vlf chorus. J. Geophys. Res. 122 (1), 324-339. 
Glauert, S. A., Horne, R. B. \& Meredith, N. P. 2014 Three-dimensional electron radiation belt simulations using the BAS Radiation Belt Model with new diffusion models for chorus, plasmaspheric hiss, and lightning-generated whistlers. J. Geophys. Res. 119, 268-289.

HSIEH, Y.-K. \& OMURA, Y. $2017 a$ Nonlinear dynamics of electrons interacting with oblique whistler mode chorus in the magnetosphere. J. Geophys. Res. 122, 675-694.

Hsien, Y.-K. \& OMURA, Y. $2017 b$ Study of wave-particle interactions for whistler mode waves at oblique angles by utilizing the gyroaveraging method. Radio Sci. 52 (10), 1268-1281.

Itin, A. P., Neishtadt, A. I. \& Vasiliev, A. A. 2000 Captures into resonance and scattering on resonance in dynamics of a charged relativistic particle in magnetic field and electrostatic wave. Physica D 141, 281-296.

KARPMAN, V. I. 1974 Nonlinear effects in the ELF waves propagating along the magnetic field in the magnetosphere. Space Sci. Rev. 16, 361-388.

Karpman, V. I., Istomin, J. N. \& ShKlyar, D. R. 1974 Nonlinear theory of a quasi-monochromatic whistler mode packet in inhomogeneous plasma. Plasma Phys. 16, 685-703.

KARpman, V. I., Istomin, J. N. \& ShKlyaR, D. R. 1975 Effects of nonlinear interaction of monochromatic waves with resonant particles in the inhomogeneous plasma. Phys. Scr. 11, 278-284.

Kennel, C. F. \& Engelmann, F. 1966 Velocity space diffusion from weak plasma turbulence in a magnetic field. Phys. Fluids 9, 2377-2388.

Landau, L. D. \& Lifshitz, E. M. 1988 Mechanics, vol. 1. Pergamon Press.

Leoncini, X., Vasiliev, A. \& Artemyev, A. 2018 Resonance controlled transport in phase space. Physica D 364, 22-26.

Le Queau, D. \& Roux, A. 1987 Quasi-monochromatic wave-particle interactions in magnetospheric plasmas. Solar Rephys. 111, 59-80.

Li, W., Bortnik, J., Thorne, R. M. \& Angelopoulos, V. 2011 a Global distribution of wave amplitudes and wave normal angles of chorus waves using THEMIS wave observations. J. Geophys. Res. 116, 12205.

Li, W., Thorne, R. M., Bortnik, J., Shprits, Y. Y., Nishimura, Y., Angelopoulos, V., Chaston, C., Le Contel, O. \& Bonnell, J. W. $2011 b$ Typical properties of rising and falling tone chorus waves. Geophys. Res. Lett. 38, L14103.

Lyons, L. R. \& Williams, D. J. 1984 Quantitative Aspects of Magnetospheric Physics. D. Reidel Publishing Company.

Ma, Q., Li, W., Thorne, R. M., Nishimura, Y., Zhang, X.-J., Reeves, G. D., Kletzing, C. A., Kurth, W. S., Hospodarsky, G. B., Henderson, M. G. et al. 2016 Simulation of energy-dependent electron diffusion processes in the Earth's outer radiation belt. J. Geophys. Res. 121, 4217-4231.

Mourenas, D., Artemyev, A. V., Agapitov, O. V., Mozer, F. S. \& Krasnoselskikh, V. V. 2016 Equatorial electron loss by double resonance with oblique and parallel intense chorus waves. J. Geophys. Res. 121, 4498-4517.

Mozer, F. S., Artemyev, A., Agapitov, O. V., Mourenas, D. \& Vasko, I. 2016 Near-relativistic electron acceleration by Landau trapping in time domain structures. Geophys. Res. Lett. 43, 508-514.

Neishtadt, A. I. 1999 On adiabatic invariance in two-frequency systems. In Hamiltonian Systems with Three or More Degrees of Freedom (ed. C. Sim), NATO ASI Series C, vol. 533, pp. 193-213. Kluwer.

Neishtadt, A. I., Petrovichev, B. A. \& Chernikov, A. A. 1989 Particle entrainment into unlimited acceleration. Sov. J. Plasma Phys. 15, 1021-1023.

NunN, D. 1971 Wave-particle interactions in electrostatic waves in an inhomogeneous medium. J. Plasma Phys. 6, 291.

NunN, D. 1974 A self-consistent theory of triggered VLF emissions. Planet. Space Sci. 22, 349-378.

NunN, D. 1986 A nonlinear theory of sideband stability in ducted whistler mode waves. Planet. Space Sci. 34, 429-451. 
NunN, D. \& OMURA, Y. 2015 A computational and theoretical investigation of nonlinear wave-particle interactions in oblique whistlers. J. Geophys. Res. 120, 2890-2911.

Omura, Y., FuruYA, N. \& Summers, D. 2007 Relativistic turning acceleration of resonant electrons by coherent whistler mode waves in a dipole magnetic field. J. Geophys. Res. 112, 6236.

Omura, Y., Miyashita, Y., Yoshikawa, M., Summers, D., Hikishima, M., Ebihara, Y. \& KubotA, Y. 2015 Formation process of relativistic electron flux through interaction with chorus emissions in the Earth's inner magnetosphere. J. Geophys. Res. 120, 9545-9562.

OMURA, Y. \& ZHAO, Q. 2012 Nonlinear pitch angle scattering of relativistic electrons by EMIC waves in the inner magnetosphere. J. Geophys. Res. 117, 8227.

OMura, Y. \& ZhaO, Q. 2013 Relativistic electron microbursts due to nonlinear pitch angle scattering by EMIC triggered emissions. J. Geophys. Res. 118, 5008-5020.

Santolík, O., Kletzing, C. A., Kurth, W. S., Hospodarsky, G. B. \& Bounds, S. R. 2014 Fine structure of large-amplitude chorus wave packets. Geophys. Res. Lett. 41, 293-299.

Schulz, M. \& Lanzerotti, L. J. 1974 Particle Diffusion in the Radiation Belts. Springer.

Shapiro, V. D. \& SAgdeEv, R. Z. 1997 Nonlinear wave-particle interaction and conditions for the applicability of quasilinear theory. Phys. Rep. 283, 49-71.

Sheeley, B. W., Moldwin, M. B., Rassoul, H. K. \& Anderson, R. R. 2001 An empirical plasmasphere and trough density model: CRRES observations. J. Geophys. Res. 106, 25631-25642.

Shklyar, D. \& Matsumoto, H. 2009 Oblique whistler-mode waves in the inhomogeneous magnetospheric plasma: resonant interactions with energetic charged particles. Surv. Geophys. 30, 55-104.

SHKLYAR, D. R. 1981 Stochastic motion of relativistic particles in the field of a monochromatic wave. Sov. Phys. JETP 53, 1197-1192.

SHKLYAR, D. R. 2011 On the nature of particle energization via resonant wave-particle interaction in the inhomogeneous magnetospheric plasma. Ann. Geophys. 29, 1179-1188.

SHKLYAR, D. R. 2017 Energy transfer from lower energy to higher-energy electrons mediated by whistler waves in the radiation belts. J. Geophys. Res. 122 (1), 640-655.

Shprits, Y. Y., Kellerman, A. C., Drozdov, A. Y., Spence, H. E., Reeves, G. D. \& Baker, D. N. 2015 Combined convective and diffusive simulations: VERB-4D comparison with 17 March 2013 Van Allen Probes observations. Geophys. Res. Lett. 42, 9600-9608.

SoloveV, V. V. \& ShKlyaR, D. R. 1986 Particle heating by a low-amplitude wave in an inhomogeneous magnetoplasma. Sov. Phys. JETP 63, 272-277.

Stix, T. H. 1962 The Theory of Plasma Waves. McGraw-Hill.

Su, Z., ZHENG, H. \& WANG, S. 2010 Three-dimensional simulation of energetic outer zone electron dynamics due to wave-particle interaction and azimuthal advection. J. Geophys. Res. 115, 6203.

SUMmERS, D. \& OMURA, Y. 2007 Ultra-relativistic acceleration of electrons in planetary magnetospheres. Geophys. Res. Lett. 34, 24205.

Summers, D., Thorne, R. M. \& Xiao, F. 1998 Relativistic theory of wave-particle resonant diffusion with application to electron acceleration in the magnetosphere. J. Geophys. Res. 103, 20487-20500.

Tao, X., Bortnik, J., Albert, J. M. \& Thorne, R. M. $2012 a$ Comparison of bounce-averaged quasi-linear diffusion coefficients for parallel propagating whistler mode waves with test particle simulations. J. Geophys. Res. 117, 10205.

Tao, X., Bortnik, J., Albert, J. M., Thorne, R. M. \& Li, W. 2013 The importance of amplitude modulation in nonlinear interactions between electrons and large amplitude whistler waves. J. Atmos. Sol.-Terr. Phys. 99, 67-72.

Tao, X., Bortnik, J., Thorne, R. M., Albert, J. M. \& Li, W. $2012 b$ Effects of amplitude modulation on nonlinear interactions between electrons and chorus waves. Geophys. Res. Lett. 39, 6102 .

Thorne, R. M., Li, W., Ni, B., Ma, Q., Bortnik, J., Chen, L., Baker, D. N., Spence, H. E., Reeves, G. D., Henderson, M. G. et al. 2013 Rapid local acceleration of relativistic radiation-belt electrons by magnetospheric chorus. Nature 504, 411-414. 
Thorne, R. M., Ni, B., Tao, X., Horne, R. B. \& Meredith, N. P. 2010 Scattering by chorus waves as the dominant cause of diffuse auroral precipitation. Nature 467, 943-946.

Trakhtengerts, V. Y. 1966 Stationary states of the Earth's outer radiation zone. Geomagn. Aeron. 6, 827-836.

Tsurutani, B. T., Falkowski, B. J., Verkhoglyadova, O. P., Pickett, J. S., Santolík, O. \& LAKHINA, G. S. 2011 Quasi-coherent chorus properties: 1 implications for wave-particle interactions. J. Geophys. Res. 116, 9210.

Turner, D. L., Lee, J. H., Claudepierre, S. G., Fennell, J. F., Blake, J. B., Jaynes, A. N., Leonard, T., Wilder, F. D., Ergun, R. E., Baker, D. N. et al. 2017 Examining coherency scales, substructure, and propagation of whistler-mode chorus elements with magnetospheric multiscale (mms). J. Geophys. Res. 122, 11201-11226.

Vasiliev, A., Neishtadt, A. \& Artemyev, A. 2011 Nonlinear dynamics of charged particles in an oblique electromagnetic wave. Phys. Lett. A 375, 3075-3079.

Vedenov, A. A., Velikhov, E. P. \& SAgdeev, R. Z. 1962 Quasilinear theory of plasma oscillations. Nucl. Fusion Suppl. 2, 465-475.

Wilson, L. B. III, Cattell, C. A., Kellogg, P. J., Wygant, J. R., Goetz, K., Breneman, A. \& KerSTEN, K. 2011 The properties of large amplitude whistler mode waves in the magnetosphere: propagation and relationship with geomagnetic activity. Geophys. Res. Lett. 38, 17107.

Yoon, P. H., Pandey, V. S. \& LeE, D.-H. 2013 Relativistic electron acceleration by oblique whistler waves. Phys. Plasmas 20 (11), 112902. 